\title{
A reduced model for electrodes-coated dielectric plates
}

\author{
Yibin $\mathrm{Fu}^{1}$, Yuxin $\mathrm{Xie}^{2}$, and Luis Dorfmann ${ }^{3}$ \\ ${ }^{1}$ School of Computing and Mathematics \\ Keele University, Staffordshire ST5 5BG, UK \\ ${ }^{2}$ Department of Mechanics \\ Tianjin University, Tianjin 300072, China \\ ${ }^{3}$ Department of Civil and Environmental Engineering \\ Tufts University, MA, USA
}

\begin{abstract}
We derive a reduced theory describing the incremental deformation of an electrodes-coated dielectric plate that takes the leading-order thickness effec$\mathrm{t}$ into account. By focusing on deformations that are symmetric with respect to the mid-plane, a power series expansion of the incremental deformation and electric field in the thickness direction is used to reduce the second variation of the total energy to an optimal form. The associated Euler-Lagrange equations are then the governing equations for the reduced model. The validity of this reduced model is verified by comparing the bifurcation condition derived from it with the two-term expansion of the exact bifurcation condition in two special cases. We compare our model with another approximate theory that recently appeared in the literature.
\end{abstract}

Keywords: Nonlinear electroelasticity, dielectric membranes, Euler-Lagrange equations, stability, bifurcation.

\section{Introduction}

Stability and bifurcation criteria of dielectric plates and membranes have received considerable attention during the last few years. Early experimental 
studies include the works by Stark and Garton [39] and Blok and LeGrand [2]. They stipulated that non-uniform thinning of an electroelastic plate is caused by microscopic imperfections in the material that experience higher than average fields, resulting in confined indentations. The intensity of the electric field ceases to be uniform, increases locally, and induces electromechanical instability and inhomogeneous deformations.

More recently, pre-stretched membranes have been investigated to quantify (i) pull-in failure which is thought to occur when the voltage reaches a maximum in uniform loading, (ii) dielectric strength failure when the electric field in the material exceeds a critical value and electric discharge occurs between the electrodes and (iii) material failure when the membrane is stretched beyond a critical value [34]. Failure due to electro-creasing and electro-cavitation as well as methods to suppress instabilities are discussed in [52].

The theory of small deformations superimposed on large deformations for a general electroelastic material is developed in [17] and used in $[18,44]$ to analyze stability of a biaxially stretched electroelastic plate. The theory [17] is used in $[6,36,1,37,38]$ to investigate multilayered dielectric composites and in [22] to study both diffuse and shear-band type modes of instability. Of interest are also the results reported in [47], where the effect of an electric actuation on the appearance of a pear-shaped configuration of an elastomer ballon and its dependence on the material models are analyzed.

Theoretical predictions of instabilities in electroelastic plates and membranes are frequently restricted to the use of the Hessian approach, where the deformation is assumed homogeneous. This approach was used in, for example, $[49,50,32,31,12,13,28,14,29,45,53,48,51,24]$. In a recent paper it is shown that marginal violation of the Hessian stability criterion corresponds exactly to the satisfaction of the bifurcation condition for localized necking [21]. This necking phenomenon is usually unstable and is quickly followed by the two-phase deformation described in [23, 49].

A simplified model to account for the initial uniform thinning followed by non-homogeneous deformation of thin electroelastic films was developed in [8]. In [9] an approach based on tension field theory was used to determine the onset of compression induced wrinkling. More recently, the same authors employed an energy approach to determine the existence of non-homogeneous equilibrium configurations in thin films $[10,11]$. See also the analysis in [15], which is concerned with wrinkling instabilities. The effect of thickness imperfections on electromechanical instabilities is evaluated in [35]. Non- 
homogeneous deformations are used in [54] to estimate the onset of pull-in instability noting that non-local effects significantly decrease the instability threshold.

Although in many applications dielectric elastomers are used as thin sheets which can be modeled by a membrane theory to leading order, there is very often a need to take the leading-order thickness effect into account in order to explain some of the phenomena observed (for instance, localized thinning). Therefore, in this paper we derive a reduced model that can be used to investigate the stability of a thin electroelastic sheet with flexible electrodes coated on the top and bottom faces. We compare our model with the approximate theory proposed in $[11,55]$.

The paper is organized as follows. Section 2 defines kinematic quantities to describe the deformation of highly deformable materials. We also summarize the basic equations of the nonlinear theory of electroelasticity and the corresponding constitutive equations. The framework governing linearized incremental deformations and electric fields superimposed on a known finitely deformed configuration is included. The problem is defined in Section 3 and the reduced model derived in Section 4. In Section 5 we specialize the associated Euler-Lagrange equations to two specific problems when the material is assumed to be a neo-Hookean dielectric. First, we consider a pure shear where the primary and incremental deformations are both plane strain and show that the bifurcation condition is consistent with the results obtained from [18]. In the second problem the stability of a thin plate subject to biaxial extension is analyzed. The associated Euler-Lagrange equations are derived and compared with the corresponding formulation in [18]. It is also shown that the derived model is different from the model proposed in $[11,55]$ even in the purely mechanical case. To understand this discrepancy, we derive in Section 6 another reduced model by starting from the same representation for the total energy functional as in [11] but using the expansion of Section 4. It is shown that this model recovers the model of $[11,55]$ under the same assumptions made in the latter papers, but is different from the model of Section 5 due to the approximation that is introduced in the electric part of the total energy. The paper is concluded with a summary and additional remarks in the final section. 


\section{Basic equations}

\subsection{Kinematics}

Consider a deformable electro-sensitive body, which in the absence of mechanical loads and electric fields is unstressed and occupies the reference configuration $\mathcal{B}_{\mathrm{r}}$ with boundary $\partial \mathcal{B}_{\mathrm{r}}$. The location of a typical material particle in $\mathcal{B}_{\mathrm{r}}$ is identified by its position vector $\mathbf{X}$ relative to some fixed origin. The body is then subject to an electric field and mechanical loads as a result of which it deforms and occupies the configuration $\mathcal{B}$ with boundary $\partial \mathcal{B}$. The corresponding deformation is described in terms of the vector function $\chi$ as $\mathbf{x}=\boldsymbol{\chi}(\mathbf{X})$, where $\mathbf{x}$ denotes the position of the particle in $\mathcal{B}$ and $\mathbf{X} \in \mathcal{B}_{\mathrm{r}}$. It is assumed that $\chi$ has sufficient regularity for the ensuing analysis.

The deformation gradient tensor $\mathbf{F}$ relative to the configuration $\mathcal{B}_{\mathrm{r}}$ is given by

$$
\mathbf{F}=\operatorname{Grad} \boldsymbol{\chi}(\mathbf{X})=\operatorname{Grad} \mathbf{x}
$$

so that $\mathrm{d} \mathbf{x}=\mathbf{F} \mathrm{d} \mathbf{X}$, where Grad is the gradient operator with respect to $\mathbf{X}$. The deformation gradient has Cartesian components $F_{i A}=\partial x_{i} / \partial X_{A}$, where $i, A \in\{1,2,3\}$. Small roman indices are associated with the configuration $\mathcal{B}$ and capital indices with the reference configuration $\mathcal{B}_{\mathrm{r}}$. Let $J$ denote the determinant of $\mathbf{F}$

$$
J=\operatorname{det} \mathbf{F}>0,
$$

which relates an infinitesimal volume element $\mathrm{d} v$ in $\mathcal{B}$ to its counterpart $\mathrm{d} V$ in $\mathcal{B}_{\mathrm{r}}$ via

$$
\mathrm{d} v=J \mathrm{~d} V .
$$

Associated with $\mathbf{F}$ are the left and right Cauchy-Green deformation tensors defined, respectively, by

$$
\mathbf{B}=\mathbf{F F}^{\mathrm{T}}, \quad \mathbf{C}=\mathbf{F}^{\mathrm{T}} \mathbf{F},
$$

where the superscript ${ }^{\mathrm{T}}$ indicates the transpose of a second-order tensor.

\subsection{The equations of nonlinear electroelasticity}

For more detailed background on nonlinear electroelasticity, we refer to the books by Landau and Lifshitz [27], Kovetz [26] and Dorfmann and Ogden [19]. 
Assuming that there are no volumetric free charges within the material, as appropriate for a dielectric material, the equations satisfied by the electric field vector $\mathbf{E}$ and the electric displacement vector $\mathbf{D}$ are

$$
\operatorname{curl} \mathbf{E}=\mathbf{0}, \quad \operatorname{div} \mathbf{D}=0 .
$$

Assume that $\mathcal{B}$ is surrounded by a vacuum within which the electric and electric displacement fields are denoted by $\mathbf{E}^{\star}$ and $\mathbf{D}^{\star}$, respectively. These satisfy the equations

$$
\operatorname{curl} \mathbf{E}^{\star}=\mathbf{0}, \quad \operatorname{div} \mathbf{D}^{\star}=0,
$$

and are connected by the standard relation $\mathbf{D}^{\star}=\varepsilon_{0} \mathbf{E}^{\star}$, where $\varepsilon_{0}$ is the electric permittivity in vacuum, [30, 20].

If there is a free surface charge $\sigma_{\mathrm{f}}$ per unit area of $\partial \mathcal{B}$ then the electric boundary conditions that have to be satisfied are

$$
\mathbf{n} \times\left(\mathbf{E}^{\star}-\mathbf{E}\right)=\mathbf{0}, \quad \mathbf{n} \cdot\left(\mathbf{D}^{\star}-\mathbf{D}\right)=\sigma_{\mathrm{f}},
$$

where $\mathbf{n}$ is the unit outward normal to $\partial \mathcal{B}$.

Throughout this paper we assume that there are no mechanical body forces, in which case the equation of equilibrium can be written in the simple form

$$
\operatorname{div} \boldsymbol{\tau}=\mathbf{0},
$$

where $\boldsymbol{\tau}$ is the so-called total Cauchy stress tensor, which incorporates electrostatic body forces. It is symmetric and satisfies the boundary condition

$$
\tau \mathbf{n}=\mathbf{t}_{\mathrm{a}}+\mathbf{t}_{\mathrm{e}}^{\star}
$$

on any part of $\partial \mathcal{B}$ where the mechanical traction $\mathbf{t}_{\mathrm{a}}$ is prescribed (per unit area) and $\mathbf{t}_{\mathrm{e}}^{\star}=\boldsymbol{\tau}_{\mathrm{e}}^{\star} \mathbf{n}$ is the Maxwell traction associated with the electrostatic Maxwell stress $\boldsymbol{\tau}_{\mathrm{e}}^{\star}$. The Maxwell traction is calculated on the outside of $\partial \mathcal{B}$ where $\boldsymbol{\tau}_{\mathrm{e}}^{\star}$ is defined by

$$
\boldsymbol{\tau}_{\mathrm{e}}^{\star}=\varepsilon_{0} \mathbf{E}^{\star} \otimes \mathbf{E}^{\star}-\frac{1}{2} \varepsilon_{0}\left(\mathbf{E}^{\star} \cdot \mathbf{E}^{\star}\right) \mathbf{I},
$$

with $\mathbf{I}$ being the identity tensor. It follows from equation (6) and the connection $\mathbf{D}^{\star}=\varepsilon_{0} \mathbf{E}^{\star}$ that $\operatorname{div} \boldsymbol{\tau}_{\mathrm{e}}^{\star}=\mathbf{0}$.

The Lagrangian forms of the electric field and electric displacement vectors are denoted by $\mathbf{E}_{\mathrm{L}}$ and $\mathbf{D}_{\mathrm{L}}$, respectively, and defined by

$$
\mathbf{E}_{\mathrm{L}}=\mathbf{F}^{\mathrm{T}} \mathbf{E}, \quad \mathbf{D}_{\mathrm{L}}=J \mathbf{F}^{-1} \mathbf{D} .
$$


These satisfy the field equations

$$
\operatorname{Curl} \mathbf{E}_{\mathrm{L}}=\mathbf{0}, \quad \operatorname{Div} \mathbf{D}_{\mathrm{L}}=0,
$$

where Curl and Div are the curl and divergence operators with respect to $\mathbf{X}$. The Lagrangian forms of the boundary conditions (7) are obtained as

$$
\left(\mathbf{F}^{\mathrm{T}} \mathbf{E}^{\star}-\mathbf{E}_{\mathrm{L}}\right) \times \mathbf{N}=\mathbf{0}, \quad\left(J \mathbf{F}^{-1} \mathbf{D}^{\star}-\mathbf{D}_{\mathrm{L}}\right) \cdot \mathbf{N}=\sigma_{\mathrm{F}},
$$

where $\mathbf{N}$ is the unit outward normal to $\partial \mathcal{B}_{\mathrm{r}}$ and $\sigma_{\mathrm{F}}$ is the free charge density per unit area of $\partial \mathcal{B}_{\mathrm{r}}$.

The appropriate transformation of $\boldsymbol{\tau}$ yields the total nominal stress tensor $\mathbf{T}$ defined by

$$
\mathbf{T}=J \mathbf{F}^{-1} \boldsymbol{\tau},
$$

which in the absence of body forces satisfies the equilibrium equation

$$
\operatorname{Div} \mathbf{T}=\mathbf{0} .
$$

The corresponding traction boundary condition can be written as

$$
\mathbf{T}^{\mathrm{T}} \mathbf{N}=\mathbf{t}_{\mathrm{A}}+\mathbf{t}_{\mathrm{E}}^{\star} \quad \text { on } \partial \mathcal{B}_{\mathrm{r}},
$$

where $\mathbf{t}_{\mathrm{A}}$ is the mechanical traction per unit area of $\partial \mathcal{B}_{\mathrm{r}}$ and $\mathbf{t}_{\mathrm{E}}^{\star}=\mathbf{T}_{\mathrm{E}}^{\star \mathrm{T}} \mathbf{N}$ with $\mathbf{T}_{\mathrm{E}}^{\star}=J \mathbf{F}^{-1} \boldsymbol{\tau}_{\mathrm{e}}^{\star}$, defined on $\partial \mathcal{B}_{\mathrm{r}}$.

\subsection{Electroelastic constitutive equations}

Following Dorfmann and Ogden [16, 20] we may describe the properties of an electroelastic material in terms of a total energy density function, either $\Omega\left(\mathbf{F}, \mathbf{E}_{\mathrm{L}}\right)$ or $\Omega^{*}\left(\mathbf{F}, \mathbf{D}_{\mathrm{L}}\right)$. Here, we focus on the former, for which the total nominal stress and the Lagrangian electric field are given for a mechanically unconstrained material by

$$
\mathbf{T}=\frac{\partial \Omega}{\partial \mathbf{F}}, \quad \mathbf{D}_{\mathrm{L}}=-\frac{\partial \Omega}{\partial \mathbf{E}_{\mathrm{L}}} .
$$

For an incompressible material these are modified to

$$
\mathbf{T}=\frac{\partial \Omega}{\partial \mathbf{F}}-p \mathbf{F}^{-1}, \quad \mathbf{D}_{\mathrm{L}}=-\frac{\partial \Omega}{\partial \mathbf{E}_{\mathrm{L}}}, \quad \text { with } \quad \operatorname{det} \mathbf{F}=1,
$$

where $p$ is a Lagrange multiplier associated with the incompressibility constraint. The corresponding expressions for $\boldsymbol{\tau}$ and $\mathbf{D}$ are then given by

$$
\boldsymbol{\tau}=\mathbf{F} \frac{\partial \Omega}{\partial \mathbf{F}}-p \mathbf{I}, \quad \mathbf{D}=-\mathbf{F} \frac{\partial \Omega}{\partial \mathbf{E}_{\mathrm{L}}} .
$$




\subsection{Incremental formulation}

In this section we summarize the equations governing linearized incremental deformations and electric fields superimposed on the known finitely deformed configuration $\mathcal{B}$. For full details, including forms of the electroelastic moduli tensors, we refer to [17]. An incremental displacement and an increment in the deformation gradient, which are denoted respectively by $\dot{\chi}$ and $\dot{\mathbf{F}}=$ Grad $\dot{\boldsymbol{\chi}}$, define the current configuration. Here and henceforth a superimposed dot represents an increment in the quantity concerned. In what follows, it is convenient to identify $\dot{\chi}$ with its Eulerian equivalent denoted by $\mathbf{u}(\mathbf{x})$. It follows that

$$
\dot{\mathbf{F}}=\mathbf{L F}, \quad \dot{J}=J \operatorname{tr} \mathbf{L}, \quad\left(\frac{\dot{\mathbf{F}}}{-\mathrm{T}}\right)=-\mathbf{L}^{\mathrm{T}} \mathbf{F}^{-\mathrm{T}},
$$

where $\mathbf{L}=\operatorname{grad} \mathbf{u}$ and $\operatorname{grad}$ is the gradient operator with respect to $\mathbf{x}$. Similarly, the increments of $\mathbf{T}, \mathbf{D}_{\mathrm{L}}, \mathbf{E}_{\mathrm{L}}$ are denoted by $\dot{\mathbf{T}}, \dot{\mathbf{D}}_{\mathrm{L}}, \dot{\mathbf{E}}_{\mathrm{L}}$ and satisfy the incremental governing equations

$$
\operatorname{Div} \dot{\mathbf{T}}=\mathbf{0}, \quad \operatorname{Div} \dot{\mathbf{D}}_{\mathrm{L}}=0, \quad \operatorname{Curl} \dot{\mathbf{E}}_{\mathrm{L}}=\mathbf{0} .
$$

From $(5)_{1}$ follows that the electric field $\mathbf{E}$ can be specified in terms of an electrostatic potential $\phi$, such that $\mathbf{E}=-\operatorname{grad} \phi$. Hence,

$$
\mathbf{E}_{\mathrm{L}}=-\mathbf{F}^{\mathrm{T}} \operatorname{grad} \phi=-\operatorname{Grad} \Phi,
$$

where

$$
\phi(\mathbf{x})=\phi(\chi(\mathbf{X}))=\Phi(\mathbf{X}) .
$$

This allows us to write the increment of the electric field as

$$
\dot{\mathbf{E}}_{\mathrm{L}}=-\mathbf{F}^{\mathrm{T}} \operatorname{grad} \dot{\phi}=-\operatorname{Grad} \dot{\Phi} .
$$

We also introduce the push forward versions of the Lagrangian variables $\dot{\mathbf{T}}$, $\dot{\mathbf{D}}_{\mathrm{L}}$ and $\dot{\mathbf{E}}_{\mathrm{L}}$, which are defined by

$$
\dot{\mathbf{T}}_{0}=J^{-1} \mathbf{F} \dot{\mathbf{T}}, \quad \dot{\mathbf{D}}_{\mathrm{L} 0}=J^{-1} \mathbf{F} \dot{\mathbf{D}}_{\mathrm{L}}, \quad \dot{\mathbf{E}}_{\mathrm{L} 0}=\mathbf{F}^{-\mathrm{T}} \dot{\mathbf{E}}_{\mathrm{L}} .
$$

The incremental form of the boundary condition (16) becomes

$$
\dot{\mathbf{T}}^{\mathrm{T}} \mathbf{N}=\dot{\mathbf{t}}_{\mathrm{A}}+J \dot{\boldsymbol{\tau}}_{\mathrm{e}}^{\star} \mathbf{F}^{-\mathrm{T}} \mathbf{N}-J \boldsymbol{\tau}_{\mathrm{e}}^{\star} \mathbf{F}^{-\mathrm{T}} \dot{\mathbf{F}}^{\mathrm{T}} \mathbf{F}^{-\mathrm{T}} \mathbf{N}+\dot{J} \boldsymbol{\tau}_{\mathrm{e}}^{\star} \mathbf{F}^{-\mathrm{T}} \mathbf{N} \quad \text { on } \partial \mathcal{B}_{\mathrm{r}}
$$

or, when updated to Eulerian form,

$$
\dot{\mathbf{T}}_{0}^{\mathrm{T}} \mathbf{n}=\dot{\mathbf{t}}_{\mathrm{A} 0}+\dot{\boldsymbol{\tau}}_{\mathrm{e}}^{\star} \mathbf{n}-\boldsymbol{\tau}_{\mathrm{e}}^{\star} \mathbf{L}^{\mathrm{T}} \mathbf{n}+(\operatorname{div} \mathbf{u}) \boldsymbol{\tau}_{\mathrm{e}}^{\star} \mathbf{n} \quad \text { on } \partial \mathcal{B} .
$$




\section{Problem formulation}

We now consider an electroelastic plate with flexible electrodes on its top and bottom faces. The plate may be deformed by the application of a potential difference to the electrodes and by mechanical traction forces. Without loss of generality, we assume that the electric potential $\phi$ takes the constant and fixed values $\Phi_{0}$ and 0 on the upper and lower surfaces, respectively. Hence, no field is generated in the surrounding space, assuming that the geometry is such that the end effects can be neglected. It follows that the total energy functional, which depends on the deformation $\chi(\mathbf{X})$ and the scalar electric potential $\Phi$, has the form

$$
\Pi\{\boldsymbol{\chi}, \Phi\}=\int_{\mathcal{B}_{\mathrm{r}}} \Omega\left(\mathbf{F}, \mathbf{E}_{\mathrm{L}}\right) \mathrm{d} V-\int_{\partial \mathcal{B}_{\mathrm{r}}} \mathbf{t}_{\mathrm{A}} \cdot \mathbf{x} \mathrm{d} S .
$$

The first variation of $\Pi$ vanishes if and only if the derived electric displacement and total nominal stress satisfy the appropriate governing equations $(12)_{2},(15)$ combined with the boundary conditions $(13)_{2}$ with $\mathbf{D}^{\star}=\mathbf{0}$ and (16) with $\mathbf{t}_{\mathrm{E}}^{\star}=\mathbf{0}$; for details we refer to $[3,4]$.

We now consider an incremental deformation $\dot{\chi}$ and expand $\Pi$ around the configuration $\mathcal{B}$ up to and including quadratic terms in $\mathbf{u}$ and $\dot{\phi}$. The linear terms in $\mathbf{u}$ and $\dot{\phi}$ must vanish since the energy functional is stationary at the configuration $\mathcal{B}$. The quadratic terms thus obtained are simply half the second variation of the total energy (28) given by

$$
\Pi_{2}=\int_{\mathcal{B}}\left(\mathcal{A}_{i j l k} L_{j i} L_{k l}+2 \mathbb{A}_{i j \mid l} L_{j i} \dot{\phi}_{, l}+\mathrm{A}_{i j} \dot{\phi}_{, i} \dot{\phi}_{, j}\right) \mathrm{d} v
$$

where $\dot{\phi}_{, i}=\partial \dot{\phi} / \partial x_{i}$ and $L_{i j}=\partial u_{i} / \partial x_{j}$. The electroelastic moduli tensors in the configuration $\mathcal{B}$ have the explicit forms

$$
\begin{gathered}
\mathcal{A}_{i j l k}=J^{-1} F_{i A} F_{l B} \frac{\partial^{2} \Omega}{\partial F_{j A} \partial F_{k B}}, \quad \mathbb{A}_{i j \mid l}=-J^{-1} F_{i A} F_{l B} \frac{\partial^{2} \Omega}{\partial F_{j A} \partial E_{\mathrm{L} B}}, \\
\mathrm{~A}_{i j}=J^{-1} F_{i A} F_{j B} \frac{\partial^{2} \Omega}{\partial E_{\mathrm{L} A} \partial E_{\mathrm{L} B}},
\end{gathered}
$$

endowed with the symmetries

$$
\mathcal{A}_{i j l k}=\mathcal{A}_{l k i j}, \quad \mathbb{A}_{i j \mid l}=\mathbb{A}_{j i \mid l}, \quad \mathrm{~A}_{i j}=\mathrm{A}_{j i} .
$$


For incompressible materials, on which we focus henceforth, $J \equiv 1$ in the above and $\mathbf{u}$ satisfies the incremental incompressibility condition

$$
\operatorname{div} \mathbf{u}=0
$$

The variables $\dot{\mathbf{T}}_{0}, \dot{\mathbf{D}}_{\mathrm{L} 0}$ and $\dot{\mathbf{E}}_{\mathrm{L} 0}$, defined in (25), have the explicit forms

$$
\begin{aligned}
\dot{T}_{0 i j} & =\mathcal{A}_{i j l k} L_{k l}+\mathbb{A}_{i j \mid l} \dot{\phi}_{, l}+p L_{i j}-\dot{p} \delta_{i j}, \\
\dot{D}_{\mathrm{L} 0 i} & =-\mathbb{A}_{j l \mid i} L_{l j}-\mathrm{A}_{i j} \dot{\phi}_{, j} .
\end{aligned}
$$

It can be shown that the first variation of $\Pi_{2}$ vanishes if and only if the following governing equations and boundary conditions are satisfied:

$$
\begin{gathered}
\operatorname{div} \dot{\mathbf{T}}_{0}=\mathbf{0}, \quad \operatorname{div} \dot{\mathbf{D}}_{\mathrm{L} 0}=0 \quad \text { in } \quad \mathcal{B}, \\
\dot{\mathbf{T}}_{0} \mathbf{n}=\mathbf{0}, \quad \dot{\phi}=0 \quad \text { on } \quad \partial \mathcal{B} .
\end{gathered}
$$

Using equations (34) and (35) in (28) yields the compact form

$$
\Pi_{2}=\int_{\mathcal{B}}\left[\operatorname{tr}\left(\dot{\mathbf{T}}_{0} \mathbf{L}\right)-\dot{\mathbf{D}}_{\mathrm{L} 0} \cdot \operatorname{grad} \dot{\phi}\right] \mathrm{d} v .
$$

\section{Reduced model}

In what follows the configuration $\mathcal{B}$ is identified by the domain

$$
\omega \times\left[-\frac{h}{2}, \frac{h}{2}\right],
$$

where $\omega$ is the mid-plane of the deformed plate and $h$ is the thickness, which is assumed small compared with a typical length scale of the deformation (e.g. the wavelength of a bifurcation mode). We introduce unit vectors $\mathbf{e}_{1}, \mathbf{e}_{2}, \mathbf{e}_{3}$ to identify the three coordinate axes, with $\mathbf{e}_{3}$ the out-of-plane direction.

The central idea is to assume a power expansion for $\mathbf{u}, \dot{\phi}$ and $\dot{p}$ in terms of $x_{3}$, and then to expand the above energy functional further, in a consistent manner, up to and including terms cubic in $h$. The associated Euler-Lagrange equations then yield an approximate theory for thin plates. Such a reduction has been carried out in [43] for a generally anisotropic, compressible and unstressed plate, and in [7] for the flexural/bending deformation of an incompressible pre-stressed plate. 
We introduce Greek subscripts and adopt the convention that they range from 1 to 2 . We assume that the mid-plane is a symmetry plane and that the pre-stress takes the form $\boldsymbol{\tau}_{\alpha \beta} \mathbf{e}_{\alpha} \otimes \mathbf{e}_{\beta}$, hence $\tau_{13}=\tau_{23}=\tau_{33}=0$. Under these assumptions, the flexural and extensional deformations are decoupled. For extensional deformations, we employ the following Taylor expansions

$$
\begin{aligned}
& \mathbf{u}=\left(\mathbf{v}+\frac{1}{2} x_{3}^{2} \mathbf{b}+\ldots\right)+\left(x_{3} a_{3}+\frac{1}{6} x_{3}^{3} c_{3}+\ldots\right) \mathbf{e}_{3}, \\
& \dot{p}=\dot{p}^{(0)}+\frac{1}{2} x_{3}^{2} \dot{p}^{(2)}+\ldots, \quad \dot{\phi}=\dot{\phi}^{(0)}+\frac{1}{2} x_{3}^{2} \dot{\phi}^{(2)}+\ldots,
\end{aligned}
$$

where the vectors $\mathbf{v}$ and $\mathbf{b}$ are functions of $x_{\alpha}$ and are perpendicular to $\mathbf{e}_{3}$, whereas $a_{3}, c_{3}, \dot{p}^{(0)}, \dot{\phi}^{(0)}, \dot{p}^{(2)}, \dot{\phi}^{(2)}, \ldots$ are all scalar functions of $x_{\alpha}$. The above forms are based on the fact that for the extensional mode under investigation, in-plane displacement, the pressure, and the electric potential should be even functions of $x_{3}$, whereas the out-of-plane displacement (i.e. the coefficient of $\mathbf{e}_{3}$ in (40)) should be an odd function of $x_{3}$. We observe that similar expansions were used in $[42,43,5,46]$, but in $[11,55]$ the authors only included the terms $\mathbf{v}+x_{3} a_{3} \mathbf{e}_{3}$ in their expression for $\mathbf{u}$. One of the aims of the present study is to assess the stability implications of this additional approximation.

In what follows, we decompose the gradient of a function $\mathbf{f}(\mathbf{x})$ as

$$
\operatorname{grad} \mathbf{f}=\frac{\partial \mathbf{f}}{\partial x_{i}} \otimes \mathbf{e}_{i}=\frac{\partial \mathbf{f}}{\partial x_{\alpha}} \otimes \mathbf{e}_{\alpha}+\frac{\partial \mathbf{f}}{\partial x_{3}} \otimes \mathbf{e}_{3}=\nabla \mathbf{f}+\frac{\partial \mathbf{f}}{\partial x_{3}} \otimes \mathbf{e}_{3},
$$

where $\nabla$ denotes the $2 \mathrm{D}$ gradient operator. Thus, the gradient of the incremental displacement vector $\mathbf{u}$ can be expressed in the convenient form

$$
\mathbf{L}=\operatorname{grad} \mathbf{u}=\mathbf{L}^{(0)}+x_{3} \mathbf{L}^{(1)}+\frac{1}{2} x_{3}^{2} \mathbf{L}^{(2)}+\ldots
$$

where

$$
\begin{aligned}
& \mathbf{L}^{(0)}=v_{\alpha, \beta} \mathbf{e}_{\alpha} \otimes \mathbf{e}_{\beta}+a_{3} \mathbf{e}_{3} \otimes \mathbf{e}_{3}, \\
& \mathbf{L}^{(1)}=a_{3, \alpha} \mathbf{e}_{3} \otimes \mathbf{e}_{\alpha}+b_{\alpha} \mathbf{e}_{\alpha} \otimes \mathbf{e}_{3}, \\
& \mathbf{L}^{(2)}=b_{\alpha, \beta} \mathbf{e}_{\alpha} \otimes \mathbf{e}_{\beta}+c_{3} \mathbf{e}_{3} \otimes \mathbf{e}_{3} .
\end{aligned}
$$

It follows from the incompressibility condition (33) that $\operatorname{div} \mathbf{L}^{(j)}=0, j=$ $0,1, \ldots$, from which we obtain

$$
a_{3}=-\operatorname{div} \mathbf{v}, \quad c_{3}=-\operatorname{div} \mathbf{b} .
$$


On substituting (40), (41) and (43) into the incremental forms (34) and (35), we obtain

$$
\begin{aligned}
\dot{T}_{0 i j} & =\dot{T}_{0 i j}^{(0)}+x_{3} \dot{T}_{0 i j}^{(1)}+\frac{1}{2} x_{3}^{2} \dot{T}_{0 i j}^{(2)}+\ldots, \\
\dot{D}_{\mathrm{L} 0 i} & =\dot{D}_{\mathrm{L} 0 i}^{(0)}+x_{3} \dot{D}_{\mathrm{L} 0 i}^{(1)}+\frac{1}{2} x_{3}^{2} \dot{D}_{\mathrm{L} 0 i}^{(2)}+\ldots,
\end{aligned}
$$

where

$$
\begin{aligned}
& \dot{T}_{0 i j}^{(0)}=\mathcal{A}_{i j l k} L_{k l}^{(0)}+p L_{i j}^{(0)}-\dot{p}^{(0)} \delta_{i j}+\mathbb{A}_{i j \mid \alpha} \dot{\phi}_{, \alpha}^{(0)}, \\
& \dot{T}_{0 i j}^{(1)}=\mathcal{A}_{i j l k} L_{k l}^{(1)}+p L_{i j}^{(1)}+\mathbb{A}_{i j \mid 3} \dot{\phi}^{(2)}, \\
& \dot{T}_{0 i j}^{(2)}=\mathcal{A}_{i j l k} L_{k l}^{(2)}+p L_{i j}^{(2)}-\dot{p}^{(2)} \delta_{i j}+\mathbb{A}_{i j \mid \alpha} \dot{\phi}_{, \alpha}^{(2)},
\end{aligned}
$$

and

$$
\begin{aligned}
\dot{D}_{\mathrm{L} 0 i}^{(0)} & =-\mathbb{A}_{j l \mid i} L_{l j}^{(0)}-\mathrm{A}_{i \alpha} \dot{\phi}_{, \alpha}^{(0)}, \\
\dot{D}_{\mathrm{L} 0 i}^{(1)} & =-\mathbb{A}_{j l \mid i} L_{l j}^{(1)}-\mathrm{A}_{i 3} \dot{\phi}^{(2)}, \\
\dot{D}_{\mathrm{L} 0 i}^{(2)} & =-\mathbb{A}_{j l \mid i} L_{l j}^{(2)}-\mathrm{A}_{i \alpha} \dot{\phi}_{, \alpha}^{(2)}
\end{aligned}
$$

The equilibrium equations (36) specialize to

$$
\begin{aligned}
& \dot{T}_{0 \beta j, \beta}^{(0)}+\dot{T}_{03 j}^{(1)}+\left(\dot{T}_{0 \beta j, \beta}^{(1)}+\dot{T}_{03 j}^{(2)}\right) x_{3}+\cdots=0, \\
& \dot{D}_{\mathrm{L} 0 \beta, \beta}^{(0)}+\dot{D}_{\mathrm{L} 03}^{(1)}+\left(\dot{D}_{\mathrm{L} 03}^{(2)}+\dot{D}_{\mathrm{L} 0 \beta, \beta}^{(1)}\right) x_{3}+\cdots=0 .
\end{aligned}
$$

Since they are valid for any $x_{3}$, it follows that

$$
\begin{aligned}
\dot{T}_{0 \beta \alpha, \beta}^{(0)}+\dot{T}_{03 \alpha}^{(1)}=0, & & \dot{T}_{0 \beta \alpha, \beta}^{(1)}+\dot{T}_{03 \alpha}^{(2)}=0, \\
\dot{D}_{\mathrm{L} 0 \beta, \beta}^{(0)}+\dot{D}_{\mathrm{L} 03}^{(1)}=0, & & \dot{D}_{\mathrm{L} 03}^{(2)}+\dot{D}_{\mathrm{L} 0 \beta, \beta}^{(1)}=0 .
\end{aligned}
$$

From $(53)_{1}$ we find that

$$
\begin{aligned}
\mathrm{A}_{33} \dot{\phi}^{(2)} & =-\mathbb{A}_{j l \mid \beta} L_{l j, \beta}^{(0)}-\mathrm{A}_{\beta \alpha} \dot{\phi}_{, \alpha \beta}^{(0)}-\mathbb{A}_{j l \mid 3} L_{l j}^{(1)}, \\
& =-\mathbb{A}_{\gamma \alpha \mid \beta} v_{\alpha, \gamma \beta}-\mathbb{A}_{33 \mid \beta} a_{3, \beta}-\mathrm{A}_{\beta \alpha} \dot{\phi}_{, \alpha \beta}^{(0)}-\mathbb{A}_{\alpha 3 \mid 3} a_{3, \alpha}-\mathbb{A}_{3 \alpha \mid 3} b_{\alpha} .
\end{aligned}
$$

The stress free boundary conditions at the top and bottom surfaces, by specializing (27) to the current problem, become

$$
\dot{T}_{03 j}=0 \quad \text { on } \quad x_{3}= \pm \frac{h}{2}
$$


and, by using (46), can be written in the equivalent form

$$
\dot{T}_{03 j}^{(0)} \pm \frac{h}{2} \dot{T}_{03 j}^{(1)}+\frac{h^{2}}{8} \dot{T}_{03 j}^{(2)}+O\left(h^{3}\right)=0 .
$$

Adding and subtracting these two boundary conditions results in

$$
\dot{T}_{03 j}^{(0)}=O\left(h^{2}\right), \quad \dot{T}_{03 j}^{(1)}=O\left(h^{2}\right),
$$

and $(52)_{1}$ gives

$$
\dot{T}_{0 \beta \alpha, \beta}^{(0)}=O\left(h^{2}\right) \text {. }
$$

By taking $j=3$ in $(57)_{1}$ and $j=\alpha$ in $(57)_{2}$, we have

$$
\begin{aligned}
& \mathcal{A}_{33 l k} L_{k l}^{(0)}+p L_{33}^{(0)}-\dot{p}^{(0)}+\mathbb{A}_{33 \mid \alpha} \dot{\phi}_{, \alpha}^{(0)}=O\left(h^{2}\right), \\
& \mathcal{A}_{3 \alpha l k} L_{k l}^{(1)}+p L_{3 \alpha}^{(1)}+\mathbb{A}_{3 \alpha \mid 3} \dot{\phi}^{(2)}=O\left(h^{2}\right),
\end{aligned}
$$

from which we deduce

$$
\begin{gathered}
\dot{p}^{(0)}=\mathcal{A}_{33 \alpha \beta} v_{\beta, \alpha}+a_{3}\left(\mathcal{A}_{3333}+p\right)+\mathbb{A}_{33 \mid \alpha} \dot{\phi}_{, \alpha}^{(0)}+O\left(h^{2}\right), \\
\mathcal{A}_{3 \alpha 3 \beta} b_{\beta}=\left(\mathcal{A}_{3 \alpha \beta 3}+p \delta_{\alpha \beta}\right)(\operatorname{div} \mathbf{v})_{, \beta}-\mathbb{A}_{3 \alpha \mid 3} \dot{\phi}^{(2)}+O\left(h^{2}\right) .
\end{gathered}
$$

Note that equation (62) can be solved simultaneously with (54) to find $\mathbf{b}$ and $\dot{\phi}^{(2)}$.

We now specialize $\Pi_{2}$, given in (38), to the reduced model. Substituting $(41)_{2},(43),(46)$ and (47) into (38) and integrating from $x_{3}=-h / 2$ to $x_{3}=h / 2$, yields

$$
\begin{aligned}
\Pi_{2}= & \int_{\omega}\left[h \dot{T}_{0 i j}^{(0)} L_{j i}^{(0)}+\frac{h^{3}}{12}\left(\frac{1}{2} \dot{T}_{0 i j}^{(0)} L_{j i}^{(2)}+\dot{T}_{0 i j}^{(1)} L_{j i}^{(1)}+\frac{1}{2} \dot{T}_{0 i j}^{(2)} L_{j i}^{(0)}\right)\right. \\
& \left.-h \dot{D}_{\mathrm{L} 0 \alpha}^{(0)} \dot{\phi}_{, \alpha}^{(0)}-\frac{h^{3}}{12}\left(\frac{1}{2} \dot{D}_{\mathrm{L} 0 \alpha}^{(0)} \dot{\phi}_{, \alpha}^{(2)}+\dot{D}_{\mathrm{L} 03}^{(1)} \dot{\phi}^{(2)}+\frac{1}{2} \dot{D}_{\mathrm{L} 0 \alpha}^{(2)} \dot{\phi}_{, \alpha}^{(0)}\right)\right] \mathrm{d} s .
\end{aligned}
$$

This can be simplified to

$$
\begin{aligned}
\Pi_{2}= & \int_{\omega}\left[h \dot{T}_{0 i j}^{(0)} L_{j i}^{(0)}-h \dot{D}_{\mathrm{L} 0 \alpha}^{(0)} \dot{\phi}_{, \alpha}^{(0)}\right. \\
& \left.+\frac{h^{3}}{12}\left(\dot{T}_{0 i j}^{(0)} L_{j i}^{(2)}+\dot{T}_{0 i j}^{(1)} L_{j i}^{(1)}-\dot{D}_{\mathrm{L} 0 \alpha}^{(0)} \dot{\phi}_{, \alpha}^{(2)}-\dot{D}_{\mathrm{L} 03}^{(1)} \dot{\phi}^{(2)}\right)\right] \mathrm{d} s,
\end{aligned}
$$


or equivalently,

$$
\begin{aligned}
\Pi_{2}= & \int_{\omega}\left[h \dot{T}_{0 \alpha \beta}^{(0)} L_{\beta \alpha}^{(0)}+h \dot{T}_{033}^{(0)} L_{33}^{(0)}-h \dot{D}_{\mathrm{L} 0 \alpha}^{(0)} \dot{\phi}_{, \alpha}^{(0)}\right. \\
& \left.+\frac{h^{3}}{12}\left(\dot{T}_{0 \alpha \beta}^{(0)} L_{\beta \alpha}^{(2)}+\dot{T}_{033}^{(0)} L_{33}^{(2)}+\dot{T}_{0 \alpha 3}^{(1)} L_{3 \alpha}^{(1)}+\dot{T}_{03 \alpha}^{(1)} L_{\alpha 3}^{(1)}-\dot{D}_{\mathrm{L} 0 \alpha}^{(0)} \dot{\phi}_{, \alpha}^{(2)}-\dot{D}_{\mathrm{L} 03}^{(1)} \dot{\phi}^{(2)}\right)\right] \mathrm{d} s .
\end{aligned}
$$

From the results shown in (59) and (60) follows that $h^{3} \dot{T}_{033}^{(0)}$ and $h^{3} \dot{T}_{03 \alpha}$ are of order $h^{5}$ and the corresponding terms can therefore be neglected. Thus,

$$
\begin{aligned}
\Pi_{2}= & \int_{\omega}\left[h \dot{T}_{0 \alpha \beta}^{(0)} v_{\beta, \alpha}+h \dot{T}_{033}^{(0)} a_{3}-h \dot{D}_{\mathrm{L} 0 \alpha}^{(0)} \dot{\phi}_{, \alpha}^{(0)}\right. \\
& \left.+\frac{h^{3}}{12}\left(\dot{T}_{0 \alpha \beta}^{(0)} b_{\beta, \alpha}+\dot{T}_{0 \alpha 3}^{(1)} a_{3, \alpha}-\dot{D}_{\mathrm{L} 0 \alpha}^{(0)} \dot{\phi}_{, \alpha}^{(2)}-\dot{D}_{\mathrm{L} 03}^{(1)} \dot{\phi}^{(2)}\right)\right] \mathrm{d} s .
\end{aligned}
$$

With the use of (58) we find that

$$
\dot{T}_{0 \alpha \beta}^{(0)} b_{\beta, \alpha}=\left(\dot{T}_{0 \alpha \beta}^{(0)} b_{\beta}\right)_{, \alpha}-\dot{T}_{0 \alpha \beta, \alpha}^{(0)} b_{\beta, \alpha}=\left(\dot{T}_{0 \alpha \beta}^{(0)} b_{\beta}\right)_{, \alpha}+O\left(h^{2}\right)
$$

and, using the divergence theorem, we obtain

$$
\int_{\omega} \dot{T}_{0 \alpha \beta}^{(0)} b_{\beta, \alpha} \mathrm{d} s=\int_{\partial \omega} \dot{T}_{0 \alpha \beta}^{(0)} b_{\beta} n_{\alpha} \mathrm{d} \gamma+O\left(h^{2}\right),
$$

where $\partial \omega$ is the edge of the mid-plane and $n_{\alpha}$ is the component of the unit normal to the mid-plane. For the case of dead loading at the edge of the plate we have $\dot{T}_{0 \alpha \beta}^{(0)} n_{\alpha}=0$ and so the integral involving $\dot{T}_{0 \alpha \beta}^{(0)} b_{\beta, \alpha}$ is of order $h^{2}$ and can be dropped from (66). Hence, we retain

$$
\begin{aligned}
\Pi_{2}^{\mathrm{d}}= & \int_{\omega}\left[h \dot{T}_{0 \alpha \beta}^{(0)} v_{\beta, \alpha}+h \dot{T}_{033}^{(0)} a_{3}-h \dot{D}_{\mathrm{L} 0 \alpha}^{(0)} \dot{\phi}_{, \alpha}^{(0)}\right. \\
& \left.+\frac{h^{3}}{12}\left(\dot{T}_{0 \alpha 3}^{(1)} a_{3, \alpha}-\dot{D}_{\mathrm{L} 0 \alpha}^{(0)} \dot{\phi}_{, \alpha}^{(2)}-\dot{D}_{\mathrm{L} 03}^{(1)} \dot{\phi}^{(2)}\right)\right] \mathrm{d} s,
\end{aligned}
$$

where the superscript ${ }^{\mathrm{d}}$ is added to signify the fact that the expression is associated with the dead-load case. 
For convenience we now write the components of the incremental stress tensor $\dot{\mathbf{T}}_{\mathbf{0}}$ in the explicit forms

$$
\begin{aligned}
\dot{T}_{0 \alpha \beta}^{(0)} & =\mathcal{A}_{\alpha \beta l k} L_{k l}^{(0)}+p L_{\alpha \beta}^{(0)}-\dot{p}^{(0)} \delta_{\alpha \beta}+\mathbb{A}_{\alpha \beta \mid \gamma} \dot{\phi}_{, \gamma}^{(0)}, \\
& =\left(\mathcal{A}_{\alpha \beta \delta \gamma}+p \delta_{\alpha \gamma} \delta_{\delta \beta}\right) v_{\gamma, \delta}-\mathcal{A}_{\alpha \beta 33} \operatorname{div} \mathbf{v}-\dot{p}^{(0)} \delta_{\alpha \beta}+\mathbb{A}_{\alpha \beta \mid \gamma} \dot{\phi}_{, \gamma}^{(0)}, \\
\dot{T}_{033}^{(0)} & =\mathcal{A}_{33 \delta \gamma} v_{\gamma, \delta}-\left(\mathcal{A}_{3333}+p\right) \operatorname{div} \mathbf{v}-\dot{p}^{(0)}+\mathbb{A}_{33 \mid \gamma} \dot{\phi}_{, \gamma}^{(0)},
\end{aligned}
$$

and

$$
\begin{aligned}
\dot{T}_{0 \alpha 3}^{(1)} & =\mathcal{A}_{\alpha 3 l k} L_{k l}^{(1)}+p L_{\alpha 3}^{(1)}+\mathbb{A}_{\alpha 3 \mid 3} \dot{\phi}^{(2)} \\
& =-\mathcal{A}_{\alpha 3 \delta 3}(\operatorname{div} \mathbf{v})_{, \delta}+\left(\mathcal{A}_{\alpha 33 \gamma}+p \delta_{\alpha \gamma}\right) b_{\gamma}+\mathbb{A}_{\alpha 3 \mid 3} \dot{\phi}^{(2)}
\end{aligned}
$$

Similarly, the components of the incremental displacement vector $\dot{\mathbf{D}}_{\mathrm{L} 0}$ become

$$
\begin{aligned}
& \dot{D}_{\mathrm{L} 0 \alpha}^{(0)}=-\mathbb{A}_{j l \mid \alpha} L_{l j}^{(0)}-\mathrm{A}_{\alpha \beta} \dot{\phi}_{, \beta}^{(0)}=-\mathbb{A}_{\gamma \beta \mid \alpha} v_{\beta, \gamma}+\mathbb{A}_{33 \mid \alpha} \operatorname{div} \mathbf{v}-\mathrm{A}_{\alpha \beta} \dot{\phi}_{, \beta}^{(0)}, \\
& \dot{D}_{\mathrm{L} 03}^{(1)}=-\mathbb{A}_{j l \mid 3} L_{l j}^{(1)}-\mathrm{A}_{33} \dot{\phi}^{(2)}=-\mathbb{A}_{3 \alpha \mid 3} b_{\alpha}+\mathbb{A}_{\alpha 3 \mid 3}(\operatorname{div} \mathbf{v})_{, \alpha}-\mathrm{A}_{33} \dot{\phi}^{(2)} .
\end{aligned}
$$

It follows that

$$
\begin{aligned}
& \dot{T}_{0 \alpha \beta}^{(0)} v_{\beta, \alpha}+\dot{T}_{033}^{(0)} a_{3}-\dot{D}_{\mathrm{L} 0 \alpha}^{(0)} \dot{\phi}_{, \alpha}^{(0)} \\
& =\left(\mathcal{A}_{\alpha \beta \delta \gamma}+p \delta_{\alpha \gamma} \delta_{\delta \beta}\right) v_{\gamma, \delta} v_{\beta, \alpha}+2 \mathcal{A}_{\alpha \beta 33} v_{\beta, \alpha} a_{3}+\left(\mathcal{A}_{3333}+p\right) a_{3}^{2} \\
& +2 \mathbb{A}_{\alpha \beta \mid \gamma} \dot{\phi}_{, \gamma}^{(0)} v_{\beta, \alpha}+2 a_{3} \mathbb{A}_{33 \mid \gamma} \dot{\phi}_{, \gamma}^{(0)}+\mathrm{A}_{\alpha \beta} \dot{\phi}_{, \beta}^{(0)} \dot{\phi}_{, \alpha}^{(0)},
\end{aligned}
$$

where use has been made of the relation $\operatorname{tr} \mathbf{L}^{(0)}=v_{\alpha, \alpha}+a_{3}=0$ to eliminate the term involving $\dot{p}^{(0)}$. Thus, the evaluation of the $O(h)$ term in (69) does not involve $\dot{p}^{(0)}$, hence

$$
\begin{aligned}
\Pi_{2}^{\mathrm{d}}= & h \int_{\omega}\left\{\left(\mathcal{A}_{\alpha \beta \delta \gamma}+p \delta_{\alpha \gamma} \delta_{\delta \beta}\right) v_{\gamma, \delta} v_{\beta, \alpha}+2 \mathcal{A}_{\alpha \beta 33} v_{\beta, \alpha} a_{3}+\left(\mathcal{A}_{3333}+p\right) a_{3}^{2}\right. \\
& +2 \mathbb{A}_{\alpha \beta \mid \gamma} \dot{\phi}_{, \gamma}^{(0)} v_{\beta, \alpha}+2 \mathbb{A}_{33 \mid \gamma} \dot{\phi}_{, \gamma}^{(0)} a_{3}+\mathrm{A}_{\alpha \beta} \dot{\phi}_{, \beta}^{(0)} \dot{\phi}_{, \alpha}^{(0)}+\frac{h^{2}}{12}\left[\mathcal{A}_{\alpha 3 \delta 3} a_{3, \delta} a_{3, \alpha}\right. \\
& \left.\left.+\left(\mathcal{A}_{\alpha 33 \gamma}+p \delta_{\alpha \gamma}\right) b_{\gamma} a_{3, \alpha}+\mathbb{A}_{\alpha 3 \mid 3} \dot{\phi}^{(2)} a_{3, \alpha}-\dot{D}_{\mathrm{L} 0 \alpha}^{(0)} \dot{\phi}_{, \alpha}^{(2)}-\dot{D}_{\mathrm{L} 03}^{(1)} \dot{\phi}^{(2)}\right]\right\} \mathrm{d} s .
\end{aligned}
$$


The quantities $\dot{\phi}^{(2)}$ and $b_{\gamma}$ are determined by solving simultaneously equations (54) and (62), which are copied here for ease of reference

$$
\begin{aligned}
\mathrm{A}_{33} \dot{\phi}^{(2)}+\mathbb{A}_{3 \alpha \mid 3} b_{\alpha} & =-\mathbb{A}_{\gamma \alpha \mid \beta} v_{\alpha, \gamma \beta}-\mathbb{A}_{33 \mid \beta} a_{3, \beta}-\mathrm{A}_{\beta \alpha} \dot{\phi}_{, \alpha \beta}^{(0)}-\mathbb{A}_{\alpha 3 \mid 3} a_{3, \alpha} \\
\mathbb{A}_{3 \alpha \mid 3} \dot{\phi}^{(2)}+\mathcal{A}_{3 \alpha 3 \gamma} b_{\gamma} & =\left(\mathcal{A}_{3 \alpha \beta 3}+p \delta_{\alpha \beta}\right)(\operatorname{div} \mathbf{v})_{, \beta} .
\end{aligned}
$$

The Euler-Lagrange equations associated with the functional (70) become the governing equations for our reduced model. They can be derived in a straightforward manner even for a general material model, but they are quite involved and are hence not written out for the sake of brevity. However, in the next section, these governing equations will be given for a special choice of the free energy function.

\section{Validation and comparison with the model in [11]}

In the following we consider an energy function $\Omega\left(\mathbf{F}, \mathbf{E}_{\mathrm{L}}\right)$ that is additively decomposed in a purely mechanical contribution and a part associated with the electric field. We consider the special case of an incompressible material with the mechanical energy given by the neo-Hookean model and the electric contribution by an isotropic constitutive formulation with constant permittivity $\varepsilon$. This implies that the electric displacement $\mathbf{D}$ is connected to the electric field $\mathbf{E}$ by $\mathbf{D}=\varepsilon \mathbf{E}$ such that the electroelastic energy has the form $\varepsilon \mathbf{E} \cdot \mathbf{E} / 2$. Therefore, using $(11)_{1}$, we obtain

$$
\Omega\left(\mathbf{F}, \mathbf{E}_{\mathrm{L}}\right)=\frac{1}{2} \mu\left(I_{1}-3\right)-\frac{1}{2} \varepsilon \mathbf{E}_{\mathrm{L}} \cdot \mathbf{C}^{-1} \mathbf{E}_{\mathrm{L}}
$$

where $\mu$ is the shear modulus of the material in the reference configuration.

It follows from $(11)_{1},(5)$ and $(7)_{2}$ that

$$
\begin{aligned}
\int_{\mathcal{B}_{\mathrm{r}}} \varepsilon \mathbf{E}_{\mathrm{L}} \cdot \mathbf{C}^{-1} \mathbf{E}_{\mathrm{L}} \mathrm{d} V & =-\int_{\mathcal{B}} \mathbf{D} \cdot \operatorname{grad} \phi \mathrm{d} v=-\int_{\mathcal{B}} \operatorname{div}(\phi \mathbf{D}) \mathrm{d} v \\
= & -\int_{\partial \mathcal{B}} \phi \mathbf{D} \cdot \mathbf{n} \mathrm{d} a=\Phi_{0} Q,
\end{aligned}
$$

where $\mathrm{d} a$ and $\mathrm{d} v$ denote the surface and volume elements in the configuration $\mathcal{B}, \Phi_{0}$ is defined at the beginning of Section 3, and $Q$ denotes the total charge on the upper plate surface. Thus, the total energy (28) can also be written as

$$
\Pi=\int_{\mathcal{B}_{\mathrm{r}}} \frac{1}{2} \mu\left(I_{1}-3\right) \mathrm{d} V-\int_{\partial \mathcal{B}_{\mathrm{r}}} \mathbf{t}_{\mathrm{A}} \cdot \mathbf{x} \mathrm{d} S-\frac{1}{2} \Phi_{0} Q .
$$


For what follows it is useful to recall the connections

$$
\frac{\partial F_{A j}^{-1}}{\partial F_{m D}}=-F_{A m}^{-1} F_{D j}^{-1}, \quad \frac{\partial C_{D E}^{-1}}{\partial F_{j A}}=-C_{A E}^{-1} F_{D j}^{-1}-C_{D A}^{-1} F_{E j}^{-1} .
$$

The specialized forms of electroelastic moduli tensors (30) and (31) are

$$
\mathcal{A}_{i j l k}=\mu \delta_{j k} B_{i l}-\varepsilon\left(E_{j} E_{k} \delta_{i l}+E_{j} E_{l} \delta_{i k}+E_{i} E_{k} \delta_{j l}\right)
$$

and

$$
\mathbb{A}_{i j \mid l}=\varepsilon\left(E_{j} \delta_{i l}+E_{i} \delta_{j l}\right), \quad \mathrm{A}_{i j}=-\varepsilon \delta_{i j} .
$$

Using (19) we find that the total Cauchy stress is given by

$$
\boldsymbol{\tau}=\mu \mathbf{B}+\varepsilon \mathbf{E} \otimes \mathbf{E}-p \mathbf{I} .
$$

\subsection{Pure shear}

We first consider the case when the primary and incremental deformations are both plane-strain. The deformation gradient tensor of the configuration $\mathcal{B}$ relative to $\mathcal{B}_{\mathrm{r}}$ is given by

$$
\mathbf{F}=\lambda \mathbf{e}_{1} \otimes \mathbf{e}_{1}+\mathbf{e}_{2} \otimes \mathbf{e}_{2}+\lambda^{-1} \mathbf{e}_{3} \otimes \mathbf{e}_{3},
$$

where $\mathbf{e}_{3}$ again identifies the out of plane direction. It follows that the incremental quantities defined in (40) and (41) are independent of $x_{2}$ and that the component $v_{2}$ of the vector $\mathbf{v}$ vanishes.

The electric field $\mathbf{E}$ in the material, which is generated by a potential difference between the flexible membranes, has the nonzero component $E_{3}$ with the corresponding Lagrangian form $E_{\mathrm{L} 3}=\lambda^{-1} E_{3}$. The out of plane component of the total stress $\boldsymbol{\tau}$ vanishes and (79) gives

$$
p=\mu \lambda^{-2}+\varepsilon E_{3}^{2} .
$$

It follows that the associated Lagrangian components of the electric displacement and the total nominal stress (14) in the 1-direction are

$$
D_{\mathrm{L} 3}=\varepsilon \lambda^{2} E_{\mathrm{L} 3}, \quad T_{11}=\mu\left(\lambda-\lambda^{-3}\right)-\varepsilon \lambda E_{\mathrm{L} 3}^{2} .
$$

Hence, the functional (70) reduces to

$$
\begin{aligned}
\Pi_{2}^{\mathrm{d}} & =h \int_{\omega}\left\{\left(\mu \lambda^{2}+3 \mu \lambda^{-2}-\varepsilon E_{3}^{2}\right) v_{1}^{\prime 2}-\varepsilon \dot{\phi}^{2}\right. \\
& \left.+\frac{h^{2}}{12}\left(\mu \lambda^{2}-\mu \lambda^{-2}-\varepsilon E_{3}^{2}\right) v_{1}^{\prime \prime 2}-\varepsilon \dot{\phi}^{\prime \prime 2}+\varepsilon \dot{\phi}^{\prime} \dot{\phi}^{\prime \prime \prime}\right\} \mathrm{d} s
\end{aligned}
$$


where the superscript ' is used to denoted the derivative with respect to $x_{1}$ and equation (72) results in $b_{1}=v_{1}^{\prime \prime}$.

The associated Euler-Lagrange equations are

$$
\begin{gathered}
\dot{\phi}^{\prime \prime}-\frac{h^{2}}{6} \dot{\phi}^{(4)}=0, \\
\left(\lambda^{2}+3 \lambda^{-2}-\bar{\varepsilon} E_{3}^{2}\right) v_{1}^{\prime \prime}-\frac{h^{2}}{12}\left(\lambda^{2}-\lambda^{-2}-\bar{\varepsilon} E_{3}^{2}\right) v_{1}^{(4)}=0,
\end{gathered}
$$

where $\bar{\varepsilon}=\varepsilon / \mu$. On substituting a buckling solution of the form $v_{1}=\mathrm{e}^{i k x_{1}}$ into (85), we obtain

$$
3+\lambda^{4}-\bar{\varepsilon} E_{3}^{2} \lambda^{2}-\frac{1}{3}(k h)^{2}=0,
$$

where the leading-order result $\bar{\varepsilon} E_{3}^{2}=\lambda^{2}+3 \lambda^{-2}$ is used to eliminate $\bar{\varepsilon} E_{3}^{2}$ from the $O\left(h^{2}\right)$ term.

We next compare the result (86) with the exact bifurcation condition presented in [18]. Their incremental equations (99) and (100) give a $6 \times 6$ matrix, the determinant of which can be factorized into two factors. The two factors correspond to the extensional and flexural modes, respectively. On expanding the factor corresponding to the extensional mode in terms of $k h$ and setting the result to zero, we obtain

$$
3+\lambda^{4}-\bar{\varepsilon} E_{3}^{2} \lambda^{2}+K_{1}(k h)^{2}+O\left(h^{4}\right)=0,
$$

where

$$
K_{1}=\frac{1}{6}\left[10+9 \lambda^{2}+7 \lambda^{4}+3 \lambda^{6}+\lambda^{8}-\bar{\varepsilon} E_{3}^{2} \lambda^{2}\left(4+3 \lambda^{2}+\lambda^{4}\right)\right] .
$$

It follows from (87) that $\bar{\varepsilon} E_{3}^{2}=\lambda^{2}+3 \lambda^{-2}+O\left(h^{2}\right)$. With the use of this result, the bifurcation condition $(87)$ can be reduced to

$$
3+\lambda^{4}-\bar{\varepsilon} E_{3}^{2} \lambda^{2}-\frac{1}{3}(k h)^{2}+O\left(h^{4}\right)=0,
$$

which is consistent with (86).

We observe that in the limit $k h \rightarrow 0$, equation (88) yields $\bar{\varepsilon} E_{3}^{2} \lambda^{2}=3+\lambda^{4}$, and $(82)_{2}$ then gives $T_{11}=-4 \mu \lambda^{-3}$ which is negative. This means that the bifurcation condition cannot be satisfied when the dead-load is tensile. However, this is a consequence of the specific constitutive assumption (73). We may easily construct a material model for which the bifurcation condition (88) with $k h=0$ can be satisfied when the dead-load is tensile. 


\subsection{Bi-axial extension}

In this subsection we consider planar bi-axial extension with associated deformation gradient

$$
\mathbf{F}=\lambda \mathbf{e}_{1} \otimes \mathbf{e}_{1}+\lambda \mathbf{e}_{2} \otimes \mathbf{e}_{2}+\lambda^{-2} \mathbf{e}_{3} \otimes \mathbf{e}_{3}
$$

The electric field $\mathbf{E}$ in the material is again generated by a potential difference between flexible membranes attached to the on the top and bottom surfaces with components $E_{1}=E_{2}=0$ and $E_{3}=\lambda^{2} E_{\mathrm{L} 3}$. There are no mechanical traction applied at the top or bottom surface, and therefore the out of plane component of the total stress $\boldsymbol{\tau}$ vanishes. From (79) follows that

$$
p=\mu \lambda^{-4}+\varepsilon E_{3}^{2},
$$

and hence the non-zero components of the total Cauchy stress are

$$
\tau \equiv \tau_{11}=\tau_{22}=\mu\left(\lambda^{2}-\lambda^{-4}\right)-\varepsilon E_{3}^{2},
$$

which corresponds to the results shown by equation (105) in [18]. Relation (91) may also be compared with equation (54) in [11].

We now specialize the functional (70) to biaxial extension to obtain

$$
\begin{aligned}
\Pi_{2}^{\mathrm{d}} & =\mu h \int_{\omega}\left\{\lambda^{2} \operatorname{tr}\left(\nabla \mathbf{v} \nabla \mathbf{v}^{T}\right)+\left(\lambda^{-4}+\bar{\varepsilon} E_{3}^{2}\right) \operatorname{tr}(\nabla \mathbf{v})^{2}+2\left(\lambda^{-4}-\bar{\varepsilon} E_{3}^{2}\right) a_{3}^{2}-\bar{\varepsilon}\left|\nabla \dot{\phi}^{(0)}\right|^{2}\right. \\
& \left.+\frac{h^{2}}{12}\left[\left(\lambda^{2}-\lambda^{-4}-\bar{\varepsilon} E_{3}^{2}\right)\left|\nabla a_{3}\right|^{2}+\bar{\varepsilon}\left(\left(\nabla \dot{\phi}^{(0)}\right)^{T} \nabla \Delta \dot{\phi}^{(0)}-\left(\Delta \dot{\phi}^{(0)}\right)^{2}\right)\right]\right\} \mathrm{d} s, \quad(92)
\end{aligned}
$$

where use has been made of the equations

$$
\mathbf{b}=\nabla(\operatorname{div} \mathbf{v})=-\nabla a_{3}, \quad \dot{\phi}^{(2)}=-\Delta \dot{\phi}^{(0)},
$$

derived from (71) and (72), $\Delta$ is the 2D Laplace operator, and $\nabla \mathbf{v}^{\mathrm{T}}$ stands for $(\nabla \mathbf{v})^{\mathrm{T}}$. The associated Euler-Lagrange equations are

$$
\lambda^{2} \Delta \mathbf{v}+\left(3 \lambda^{-4}-\bar{\varepsilon} E_{3}^{2}\right) \nabla \operatorname{div} \mathbf{v}-\frac{h^{2}}{12}\left(\lambda^{2}-\lambda^{-4}-\bar{\varepsilon} E_{3}^{2}\right) \nabla \Delta \operatorname{div} \mathbf{v}=\mathbf{0}
$$

and

$$
\Delta\left(\dot{\phi}^{(0)}-\frac{h^{2}}{6} \Delta \dot{\phi}^{(0)}\right)=0
$$


We note that the equations for $\mathbf{v}$ and $\dot{\phi}^{(0)}$ are decoupled.

To compare with the bifurcation condition given in [18], we specialize (94) to plane strain deformation to obtain

$$
\left(\lambda^{2}+3 \lambda^{-4}-\bar{\varepsilon} E_{3}^{2}\right) v_{1,11}-\frac{h^{2}}{12}\left(\lambda^{2}-\lambda^{-4}-\bar{\varepsilon} E_{3}^{2}\right) v_{1,1111}+O\left(h^{4}\right)=0 .
$$

Looking for a buckling solution of the form $v_{1}=\mathrm{e}^{i k x_{1}}$, we obtain

$$
\left(\lambda^{2}+3 \lambda^{-4}-\bar{\varepsilon} E_{3}^{2}\right)+\frac{1}{12}(k h)^{2}\left(\lambda^{2}-\lambda^{-4}-\bar{\varepsilon} E_{3}^{2}\right)+O\left(h^{4}\right)=0 .
$$

On replacing $\bar{\varepsilon} E_{3}^{2}$ by the leading-order approximation $\lambda^{2}+3 \lambda^{-4}$ in the order $(k h)^{2}$ term, we obtain

$$
\lambda^{6}+3-\bar{\varepsilon} E_{3}^{2} \lambda^{4}-\frac{1}{3}(k h)^{2}+O\left(h^{4}\right)=0 .
$$

On the other hand, on expanding the bifurcation condition in [18] for the extensional mode in terms of $k h$, we obtain

$$
3+\lambda^{6}-\bar{\varepsilon} E_{3}^{2} \lambda^{4}+K_{2}(k h)^{2}+O\left(h^{4}\right)=0,
$$

where

$$
K_{2}=\frac{1}{6}\left[10+9 \lambda^{3}+7 \lambda^{6}+3 \lambda^{9}+\lambda^{12}-\bar{\varepsilon} E_{3}^{2} \lambda^{4}\left(4+3 \lambda^{3}+\lambda^{6}\right)\right] .
$$

Without compromising the accuracy of the first two terms, the above bifurcation condition (99) can be reduced to

$$
3+\lambda^{6}-\bar{\varepsilon} E_{3}^{2} \lambda^{4}-\frac{1}{3}(k h)^{2}+O\left(h^{4}\right)=0,
$$

which is consistent with (98).

We observe that in the limit $k h \rightarrow 0$, equation (100) yields $\bar{\varepsilon} E_{3}^{2} \lambda^{4}=3+\lambda^{6}$, and (91) then gives $\tau_{11}=-4 \mu \lambda^{-4}$ which is negative. As in the case of pure shear, this is a consequence of the specific constitutive assumption (73), and it is possible to find material models for which the bifurcation condition can be satisfied when the dead-load is tensile. 


\subsection{Comparison with the model in [11]}

For the case when the primary deformation is an equi-biaxial extension, De Tommasi et al. [11] derived a reduced plate model using a different procedure. Under the additional assumption that the incremental deformation is polar axisymmetric so that $\nabla \mathbf{v}=(\nabla \mathbf{v})^{\mathrm{T}}$, they obtained an equation for $\mathbf{v}$, which, in our current notation, takes the form

$$
\Gamma_{1}(\lambda) \nabla \operatorname{div} \mathbf{v}+\Gamma_{2}(\lambda) \nabla \Delta \operatorname{div} \mathbf{v}=\mathbf{0},
$$

where

$$
\Gamma_{1}(\lambda)=\lambda^{3}+3 \lambda^{-3}-\frac{\lambda \bar{\varepsilon} \Phi_{0}^{2}}{h^{2}}, \quad \Gamma_{2}(\lambda)=\frac{h^{2}}{12}\left(\frac{3 \lambda \bar{\varepsilon} \Phi_{0}^{2}}{2 h^{2}}-\lambda^{3}\right) .
$$

Under the same assumptions, our equation (94) reduces to

$$
\Gamma_{1}(\lambda) \nabla \operatorname{div} \mathbf{v}+\frac{h^{2}}{12}\left(\frac{\lambda \bar{\varepsilon} \Phi_{0}^{2}}{h^{2}}-\lambda^{3}+\lambda^{-3}\right) \nabla \Delta \operatorname{div} \mathbf{v}=\mathbf{0} .
$$

This is seen to be different from (101), even in the purely mechanical case when $\Phi_{0}=0$. Interestingly, with the use of (91) the above equation can be rewritten in the form

$$
\Gamma_{1}(\lambda) \nabla \operatorname{div} \mathbf{v}-\frac{\lambda h^{2} \tau}{12 \mu} \nabla \Delta \operatorname{div} \mathbf{v}=\mathbf{0} .
$$

For each fixed nominal stress $\tau / \lambda$, the equilibrium equation (91) defines the electric potential $\Phi_{0}$ as a function of the stretch $\lambda$. We may refer to its graph in the $\left(\lambda, \Phi_{0}\right)$-plane as the loading path. It was observed in [11] that equation (101) admits a non-trivial solution if $\Gamma_{2} / \Gamma_{1}>0$ (which may be replaced by $\Gamma_{2}>0$ since $\Gamma_{1}$ is positive in the domain of interest), and that this inequality may be satisfied along any loading path with $\tau$ positive. This means that a bifurcation may take place for all positive values of $\tau$. However, based on our model equation (103), the above inequality can never be satisfied along any loading path with positive $\tau$. Therefore, our model excludes the type of bifurcation identified in [11] when $\tau$ is positive.

\section{Further evaluation of the model in [11]}

In order to understand why our current model is different from the model developed in [11], we now analyze the same energy functional as in [11], 
namely,

$$
\Pi=\frac{1}{2} \mu \int_{\mathcal{B}_{\mathrm{r}}}\left(\tilde{I}_{1}-3\right) \mathrm{d} V-\int_{\partial \mathcal{B}_{\mathrm{r}}} \mathbf{t}_{\mathrm{A}} \cdot \tilde{\mathbf{x}} \mathrm{d} S-\frac{1}{2} \Phi_{0} \tilde{Q}
$$

where $\tilde{I}_{1}=\operatorname{tr} \tilde{\mathbf{B}}, \tilde{\mathbf{x}}$ is the position vector of a representative particle in the current configuration $\tilde{\mathcal{B}}$ that is perturbed from the finitely deformed configuration $\mathcal{B}$, and $\tilde{Q}$ is the total charge on the upper surface. It is seen that (104) has the same form as (75) except that the configuration $\mathcal{B}$ is now replaced by $\tilde{\mathcal{B}}$. Thus, the expression (104) is exact under the assumption that the total energy can be decomposed into a mechanical part given by the neo-Hookean model and an electric contribution corresponding to constant permittivity.

In $[11,55]$ the total charge $\tilde{Q}$ is computed according to the approximation

$$
\tilde{Q}=\varepsilon \int_{\tilde{S}} \frac{\Phi_{0}}{\tilde{h}} \mathrm{~d} \tilde{a},
$$

where $\tilde{h}$ denotes the plate thickness in the configuration $\tilde{\mathcal{B}}$, and $\tilde{S}$ the upper surface. We shall adopt this same assumption, but use the full expansion (40) for $\mathbf{u}$.

Consider an incremental deformation described by the vector function $\dot{\chi}$, which is superimposed on the configuration $\mathcal{B}$. The corresponding deformation gradient tensor, denoted $\tilde{\mathbf{F}}$, relative to the reference configuration $\mathcal{B}_{\text {r }}$ has the form

$$
\tilde{\mathbf{F}}=(\mathbf{I}+\mathbf{L}) \mathbf{F},
$$

where we recall that $\mathbf{L}$ is the gradient of the incremental displacement vector $\mathbf{u}$ and $\mathbf{F}$ is the deformation gradient of the configuration $\mathcal{B}$ relative to $\mathcal{B}_{\mathrm{r}}$. Equation $(4)_{1}$ gives the corresponding left Cauchy-Green tensor

$$
\tilde{\mathbf{B}}=\mathbf{B}+\mathbf{B L}^{\mathrm{T}}+\mathbf{L B}+\mathbf{L B L}^{\mathrm{T}},
$$

which, when combined with (43), becomes

$$
\tilde{\mathbf{B}}=\mathbf{B}+\mathbf{B L}^{(0) \mathrm{T}}+\mathbf{L}^{(0)} \mathbf{B}+\mathbf{L}^{(0)} \mathbf{B} \mathbf{L}^{(0) \mathrm{T}}+\mathbf{M}_{1} x_{3}+\mathbf{M}_{2} x_{3}^{2} \ldots
$$

The $\mathbf{M}_{1}$ in the above equation will not appear in our subsequent analysis, while $\mathbf{M}_{2}$ is given by

$$
\mathbf{M}_{2}=\frac{1}{2}\left(\mathbf{B} \mathbf{L}^{(2) \mathrm{T}}+\mathbf{L}^{(2)} \mathbf{B}+\mathbf{L}^{(0)} \mathbf{B} \mathbf{L}^{(2) \mathrm{T}}+\mathbf{L}^{(2)} \mathbf{B} \mathbf{L}^{(0) \mathrm{T}}+2 \mathbf{L}^{(1)} \mathbf{B} \mathbf{L}^{(1) \mathrm{T}}\right)
$$


If the primary deformation is an equi-biaxial extension with stretch $\lambda$, we then have

$$
\begin{aligned}
\operatorname{tr}\left(\mathbf{L}^{(0)} \mathbf{B}\right)= & \lambda^{2} \operatorname{div} \mathbf{v}+\lambda^{-4} a_{3}, \quad \operatorname{tr}\left(\mathbf{L}^{(0)} \mathbf{B} \mathbf{L}^{(0) \mathrm{T}}\right)=\lambda^{2} \operatorname{tr}\left[\nabla \mathbf{v} \nabla \mathbf{v}^{\mathrm{T}}\right]+\lambda^{-4} a_{3}^{2}, \\
\operatorname{tr}\left(\mathbf{B} \mathbf{L}^{(2) \mathrm{T}}\right)= & \lambda^{2} \operatorname{div} \mathbf{b}+\lambda^{-4} c_{3}, \quad \operatorname{tr}\left(\mathbf{L}^{(0)} \mathbf{B} \mathbf{L}^{(2) \mathrm{T}}\right)=\lambda^{2} \operatorname{tr}\left[\nabla \mathbf{v} \nabla \mathbf{b}^{\mathrm{T}}\right]+\lambda^{-4} a_{3} c_{3}, \\
& \operatorname{tr}\left(\mathbf{L}^{(1)} \mathbf{B} \mathbf{L}^{(1) \mathrm{T}}\right)=\lambda^{2}\left|\nabla a_{3}\right|^{2}+\lambda^{-4}|\mathbf{b}|^{2},
\end{aligned}
$$

where we recall that $\nabla$ is the $2 \mathrm{D}$ gradient operator. The expressions of $a_{3}$ and $c_{3}$ are again obtained from the incompressibility requirement of the incremental deformation, i.e. $\operatorname{det}(\mathbf{I}+\mathbf{L})=1$. Thus,

$$
\begin{gathered}
a_{3}=-\operatorname{div} \mathbf{v}+\frac{1}{2}(\operatorname{div} \mathbf{v})^{2}+\frac{1}{2} \operatorname{tr}(\nabla \mathbf{v})^{2}+\ldots, \\
c_{3}=-\operatorname{div} \mathbf{b}+2 \mathbf{b}^{\mathrm{T}} \nabla a_{3}+\operatorname{tr}(\nabla \mathbf{v} \nabla \mathbf{b})+\operatorname{div} \mathbf{v} \operatorname{div} \mathbf{b}+\ldots,
\end{gathered}
$$

where details are given in Appendix A.

The expression of the mechanical part of the total energy (104), keeping the terms quadratic in the incremental displacement, has the form

$$
\begin{aligned}
& h\left\{\lambda^{-4}\left[2(\operatorname{div} \mathbf{v})^{2}+\operatorname{tr}(\nabla \mathbf{v})^{2}\right]+\lambda^{2} \operatorname{tr}\left(\nabla \mathbf{v} \nabla \mathbf{v}^{\mathrm{T}}\right)\right\}+\frac{h^{3}}{12 \lambda^{4}}\left\{-2 \mathbf{b}^{\mathrm{T}} \nabla \operatorname{div} \mathbf{v}\right. \\
& \left.+\operatorname{tr}[\nabla \mathbf{v} \nabla \mathbf{b}]+2 \operatorname{div} \mathbf{v} \operatorname{div} \mathbf{b}+\lambda^{6} \operatorname{tr}\left[\nabla \mathbf{v} \nabla \mathbf{b}^{\mathrm{T}}\right]+\lambda^{6}|\nabla \operatorname{div} \mathbf{v}|^{2}+|\mathbf{b}|^{2}\right\} .
\end{aligned}
$$

To expand the third term on the right hand side of (104), we write

$$
\frac{1}{2} \varepsilon \Phi_{0}^{2} \int_{\tilde{S}} \frac{1}{\tilde{h}} \mathrm{~d} \tilde{a}=\frac{\varepsilon \Phi_{0}^{2}}{2 h} \int_{S} \frac{h}{\tilde{h}} \frac{\mathrm{d} \tilde{a}}{\mathrm{~d} a} \mathrm{~d} a,
$$

where $\mathrm{d} a$ and $S$ are the images of $\mathrm{d} \tilde{a}$ and $\tilde{S}$ in the configuration $\mathcal{B}$. Using the incremental deformation tensor and Nanson's formula we find that

$$
\frac{\tilde{h}}{h}=1+a_{3}, \quad \frac{\mathrm{d} \tilde{a}}{\mathrm{~d} a}=\left|(\mathbf{I}+\mathbf{L})^{-\mathrm{T}} \mathbf{e}_{3}\right| .
$$

In Appendix B it is shown that

$$
\frac{h}{\tilde{h}} \frac{\mathrm{d} \tilde{a}}{\mathrm{~d} a}=1+2 \operatorname{div} \mathbf{v}+2(\operatorname{div} \mathbf{v})^{2}-\operatorname{tr}(\nabla \mathbf{v})^{2}+\frac{h^{2}}{8}\left(\operatorname{div} \mathbf{b}+3 \operatorname{div} \mathbf{v} \operatorname{div} \mathbf{b}+|\nabla \operatorname{div} \mathbf{v}|^{2}\right) .
$$


Introducing the short-hand notation $U_{2}(\mathbf{v}, \mathbf{b})$ to define the quadratic terms in the energy functional (104) gives

$$
\Pi_{2}=\mu h \int_{S} U_{2}(\mathbf{v}, \mathbf{b}) \mathrm{d} a,
$$

where

$$
\begin{aligned}
U_{2}(\mathbf{v}, \mathbf{b}) & =\lambda^{-4}\left[2(\operatorname{div} \mathbf{v})^{2}+\operatorname{tr}(\nabla \mathbf{v})^{2}\right]+\lambda^{2} \operatorname{tr}\left(\nabla \mathbf{v} \nabla \mathbf{v}^{\mathrm{T}}\right)+\frac{h^{2}}{12 \lambda^{4}}\left\{-2 \mathbf{b}^{\mathrm{T}} \nabla \operatorname{div} \mathbf{v}\right. \\
& \left.+\operatorname{tr}[\nabla \mathbf{v} \nabla \mathbf{b}]+2 \operatorname{div} \mathbf{v} \operatorname{div} \mathbf{b}+\lambda^{6} \operatorname{tr}\left[\nabla \mathbf{v} \nabla \mathbf{b}^{\mathrm{T}}\right]+\lambda^{6}|\nabla \operatorname{div} \mathbf{v}|^{2}+|\mathbf{b}|^{2}\right\} \\
& -\frac{\bar{\varepsilon} \Phi_{0}^{2}}{h^{2}}\left[2(\operatorname{div} \mathbf{v})^{2}-\operatorname{tr}(\nabla \mathbf{v})^{2}+\frac{h^{2}}{8}\left(3 \operatorname{div} \mathbf{v} \operatorname{div} \mathbf{b}+|\nabla \operatorname{div} \mathbf{v}|^{2}\right)\right] .
\end{aligned}
$$

To recover the model equation (101), we take $\mathbf{b}=\mathbf{0}$ in (116) to obtain

$$
\begin{aligned}
U_{2}(\mathbf{v}, \mathbf{0}) & =\lambda^{-4}\left[2(\operatorname{div} \mathbf{v})^{2}+\operatorname{tr}(\nabla \mathbf{v})^{2}\right]+\lambda^{2} \operatorname{tr}\left(\nabla \mathbf{v} \nabla \mathbf{v}^{\mathrm{T}}\right)+\frac{h^{2}}{12} \lambda^{2}|\nabla \operatorname{div} \mathbf{v}|^{2} \\
& -\frac{\bar{\varepsilon} \Phi_{0}^{2}}{h^{2}}\left[2(\operatorname{div} \mathbf{v})^{2}-\operatorname{tr}(\nabla \mathbf{v})^{2}+\frac{h^{2}}{8}\left(|\nabla \operatorname{div} \mathbf{v}|^{2}\right)\right] .
\end{aligned}
$$

The associated Euler-Lagrange equation is

$$
\lambda^{3} \Delta \mathbf{v}+\left(3 \lambda^{-3}-\frac{\bar{\varepsilon} \Phi_{0}^{2} \lambda}{h^{2}}\right) \nabla \operatorname{div} \mathbf{v}+\frac{h^{2}}{12}\left(\frac{3 \bar{\varepsilon} \Phi_{0}^{2} \lambda}{2 h^{2}}-\lambda^{3}\right) \nabla \Delta \operatorname{div} \mathbf{v}=\mathbf{0} .
$$

Under the further assumption that the incremental deformation is polar axisymmetric, see the statement above equation (50) in [11], we have $\Delta \mathbf{v}=$ $\nabla$ divv, and the above equation then reduces to (101). Thus, the discrepancy between our model and the model in [11] is due to the exclusion of $\mathbf{b}$, and hence $c_{3}$ (since $c_{3}=-\operatorname{div} \mathbf{b}$, see (45)), in the expansion of $\mathbf{u}$ in [11]. Because of this exclusion, the two models are different even in the purely mechanical case when $\Phi_{0} \equiv 0$.

In order to compare with the results derived in Section 4, we substract from (116) the $O\left(h^{4}\right)$ term $h^{2} \dot{T}_{0 \alpha \beta}^{(0)} b_{\beta, \alpha} / 12$. Using equation (67), with (48), (61), (77), (78), (89) and (90), we have

$$
\begin{aligned}
\frac{h^{2}}{12 \lambda^{4}} \dot{T}_{0 \alpha \beta}^{(0)} b_{\beta, \alpha} & =\frac{h^{2}}{12 \lambda^{4}}\left[\mu \lambda^{6} \operatorname{tr}\left(\nabla \mathbf{v} \nabla \mathbf{b}^{\mathrm{T}}\right)+\left(\mu+\varepsilon \lambda^{4} E_{3}^{2}\right) \operatorname{tr}(\nabla \mathbf{v} \nabla \mathbf{b})\right. \\
& \left.+2\left(\mu-\varepsilon \lambda^{4} E_{3}^{2}\right) \operatorname{div} \mathbf{v} \operatorname{div} \mathbf{b}\right]
\end{aligned}
$$


Thus, we replace (116) by

$$
\begin{aligned}
U_{2}(\mathbf{v}, \mathbf{b}) & =\lambda^{-4}\left[2(\operatorname{div} \mathbf{v})^{2}+\operatorname{tr}(\nabla \mathbf{v})^{2}\right]+\lambda^{2} \operatorname{tr}\left(\nabla \mathbf{v} \nabla \mathbf{v}^{\mathrm{T}}\right) \\
& +\frac{h^{2}}{12 \lambda^{4}}\left(-2 \mathbf{b}^{\mathrm{T}} \nabla \operatorname{div} \mathbf{v}+\lambda^{6}|\nabla \operatorname{div} \mathbf{v}|^{2}+|\mathbf{b}|^{2}\right)-\frac{\bar{\varepsilon} \Phi_{0}^{2}}{h^{2}}\left[2(\operatorname{div} \mathbf{v})^{2}-\operatorname{tr}(\nabla \mathbf{v})^{2}\right] \\
& -\frac{\bar{\varepsilon} \Phi_{0}^{2}}{24}\left[5 \operatorname{div} \mathbf{v} \operatorname{div} \mathbf{b}+2 \operatorname{tr}(\nabla \mathbf{v} \nabla \mathbf{b})+3|\nabla \operatorname{div} \mathbf{v}|^{2}\right] .
\end{aligned}
$$

Following the approach in [42], we allow b to vary independently. Then, setting the variation of $\Pi_{2}$ with respect to $\mathbf{b}$ equal to zero gives

$$
\mathbf{b}=\left(1-\frac{7}{4} \frac{\bar{\varepsilon} \Phi_{0}^{2} \lambda^{4}}{h^{2}}\right) \nabla \operatorname{div} \mathbf{v}
$$

If we specialize the above result to the purely mechanical case by setting $\Phi_{0}=0$, we have $\mathbf{b}=\nabla$ divv. On substituting this expression back into (119), the reduced expression agrees with (92) under the same assumption. This provides a partial check on the expansions carried out in the current section.

However, our analysis in Section 5 shows that the result $\mathbf{b}=\nabla \operatorname{div} \mathbf{v}$ is also valid when electric effects are taken into account. This expression for $\mathbf{b}$ is different from (120). Obviously, the discrepancy is due to the approximation adopted in (105).

\section{Conclusion}

Reduced models for plates have enjoyed continued attention in the science community and have played an important role in helping us understand the essential features of plate response without the need to use the fully three-dimensional elasticity theory. Our current research is motivated by the observation that there exist very few reduced models for electroelastic plates and what has been achieved in the purely mechanical case has not yet been fully extended to the electroelastic case. Our current study provides a first such extension of the methodology employed by [40, 41, 42, 43], although in contrast with the purely mechanical case we have chosen not to derive the associated edge conditions; this is because in practice electrodes are rarely extended to the plate edge. The power expansion approach has also been used in the derivation of dynamic plate theories; see [25, 33] for instance. This 
approach is well-tested and, in the pure mechanical case under appropriate limits, recovers the classical Kirchhoff-Love plate theory.

In contrast, the electroelastic plate model employed by $[11,55]$ is derived using a different methodology. It involves two assumptions. Firstly, the expansion for the incremental displacement is linear in terms of the transverse coordinate $x_{3}$; it does not include the quadratic and cubic terms in (40). Secondly, the total charge that appears in the computation of the total energy is calculated with the use of the approximate formula (105). The main conclusion from our comparison is that the type of bifurcation modes described by $[11,55]$ are not predicted by the current model. To shed further light on the reduced model of $[11,55]$, we have analyzed the same energy functional as in [11] but used the full expansion (40) for the displacement. Our analysis does recover their model under appropriate assumptions, but the associated Euler-Lagrange equation is still different from the one corresponding to the model we derived, due to the approximation adopted in (105). 


\section{Appendix}

\section{A. Derivation of (110) and (111)}

The deformation gradient $\tilde{\mathbf{F}}$ relative to the the reference configuration $\mathcal{B}_{\mathrm{r}}$ is given in (106). Incompressibility of the incremental deformation requires that $\operatorname{det}(\mathbf{I}+\mathbf{L})=1$. To evaluate the determinant we use the formula

$$
\operatorname{det}(\eta \mathbf{I}-\mathbf{L})=\eta^{3}-I_{1 \mathbf{L}} \eta^{2}+I_{2 \mathbf{L}} \eta-I_{3 \mathbf{L}}
$$

where $I_{1 \mathbf{L}}, I_{2 \mathbf{L}}, I_{3 \mathbf{L}}$ are the principal invariants of $\mathbf{L}$, and $\eta$ is any scalar. In particular, taking $\eta=-1$ we find that the incompressibility condition may be written as

$$
I_{1 \mathbf{L}}+\frac{1}{2}\left(I_{1 \mathbf{L}}^{2}-\operatorname{tr} \mathbf{L}^{2}\right)+\cdots=0,
$$

where here and hereafter the dots denote cubic and higher order terms that do not participate in our analysis.

From (43) it follows that

$$
\begin{gathered}
I_{1 \mathbf{L}}=\operatorname{tr} \mathbf{L}=\operatorname{div} \mathbf{v}+a_{3}+\frac{1}{2} x_{3}^{2}\left(\operatorname{div} \mathbf{b}+c_{3}\right)+\ldots \\
\operatorname{tr} \mathbf{L}^{2}=\operatorname{tr}(\nabla \mathbf{v})^{2}+a_{3}^{2}+x_{3}^{2}\left[\operatorname{tr}(\nabla \mathbf{v} \nabla \mathbf{b})+a_{3} c_{3}+2 \mathbf{b}^{T} \nabla \mathbf{a}_{3}\right]+\cdots
\end{gathered}
$$

On substituting these expressions into (122) and equating the coefficients of $x_{3}^{0}$ and $x_{3}^{2}$ to zero, respectively, we obtain

$$
\begin{gathered}
\left(\operatorname{div} \mathbf{v}+a_{3}\right)\left\{1+\frac{1}{2}\left(\operatorname{div} \mathbf{v}+a_{3}\right)\right\}-\frac{1}{2}\left[\operatorname{tr}(\nabla \mathbf{v})^{2}+a_{3}^{2}\right]=0, \\
\left(\operatorname{div} \mathbf{b}+c_{3}\right)\left(1+\operatorname{div} \mathbf{v}+a_{3}\right)-\operatorname{tr}(\nabla \mathbf{v} \nabla \mathbf{b})-a_{3} c_{3}-2 \mathbf{b}^{2} \nabla \mathbf{a}_{3}=0 .
\end{gathered}
$$

These two exact equations are equivalent to

$$
\left(\operatorname{div} \mathbf{v}+a_{3}\right)-\frac{1}{2}\left[\operatorname{tr}(\nabla \mathbf{v})^{2}+a_{3}^{2}\right]+\cdots=0
$$

and

$$
\left(\operatorname{div} \mathbf{b}+c_{3}\right)-\operatorname{tr}(\nabla \mathbf{v} \nabla \mathbf{b})-a_{3} c_{3}-2 \mathbf{b}^{T} \nabla a_{3}+\cdots=0 .
$$

Equations (110) and (111) are obtained if $a_{3}$ is replaced by - $\operatorname{div} \mathbf{v}$ and $c_{3}$ by - divb in the quadratic terms since the errors induced are at least cubic. 


\section{B. Derivation of (114)}

In (113) $)_{2}$ Nanson's formula relates an infinitesimal small area element d $\tilde{a}$ in the current configuration to the corresponding quantity in the configuration $\mathcal{B}$. This requires the inverse of $\mathbf{I}+\mathbf{L}$ where $\mathbf{I}$ is again the identity and $\mathbf{L}$ the spacial gradient of the incremental displacement vector defined in (43). We use the decomposition

$$
\mathbf{I}+\mathbf{L}=\mathbf{K}_{1}+\frac{h}{2} \mathbf{K}_{2}=\mathbf{K}_{1}\left(\mathbf{I}+\frac{h}{2} \mathbf{K}_{1}^{-1} \mathbf{K}_{2}\right),
$$

where

$$
\begin{gathered}
\mathbf{K}_{1}=\mathbf{K}_{3}+\zeta \mathbf{e}_{3} \otimes \mathbf{e}_{3}, \quad \mathbf{K}_{2}+a_{3, \alpha} \mathbf{e}_{3} \otimes \mathbf{e}_{\alpha}+b_{\alpha} \mathbf{e}_{\alpha} \otimes \mathbf{e}_{3}, \\
\mathbf{K}_{3}=\left(\mathbf{I}-\mathbf{e}_{3} \otimes \mathbf{e}_{3}\right)+\nabla \mathbf{v}+\frac{h^{2}}{8} b_{\alpha, \beta} \mathbf{e}_{\alpha} \otimes \mathbf{e}_{\beta}, \quad \zeta=1+a_{3}+\frac{h^{2}}{8} c_{3} .
\end{gathered}
$$

The reason for this decomposition is that $\mathbf{K}_{1}$ is in block form, therefore the inverse is simply $\mathbf{K}_{1}^{-1}=\mathbf{K}_{3}^{-1}+\zeta^{-1} \mathbf{e}_{3} \otimes \mathbf{e}_{3}$. It follows that

$$
\begin{gathered}
\mathbf{K}_{1}^{-\mathrm{T}}=\mathbf{K}_{3}^{-\mathrm{T}}+\zeta^{-1} \mathbf{e}_{3} \otimes \mathbf{e}_{3}, \quad \mathbf{K}_{1}^{-\mathrm{T}} \mathbf{e}_{3}=\zeta^{-1} \mathbf{e}_{3}, \\
\mathbf{K}_{2}^{\mathrm{T}} \mathbf{K}_{1}^{-\mathrm{T}} \mathbf{e}_{3}=\zeta^{-1} \nabla a_{3}, \quad\left(\mathbf{K}_{2}^{\mathrm{T}} \mathbf{K}_{1}^{-\mathrm{T}}\right)^{2} \mathbf{e}_{3}=\zeta^{-1} \mathbf{b}^{\mathrm{T}} \mathbf{K}_{3}^{-\mathrm{T}} \nabla a_{3} \mathbf{e}_{3} .
\end{gathered}
$$

Thus,

$$
\begin{aligned}
(\mathbf{I}+\mathbf{L})^{-\mathrm{T}} & =\mathbf{K}_{1}^{-\mathrm{T}}\left[\mathbf{I}-\frac{h}{2} \mathbf{K}_{2}^{\mathrm{T}} \mathbf{K}_{1}^{-\mathrm{T}}+\frac{h^{2}}{4}\left(\mathbf{K}_{2}^{\mathrm{T}} \mathbf{K}_{1}^{-\mathrm{T}}\right)^{2}\right]+O\left(h^{2}\right), \\
(\mathbf{I}+\mathbf{L})^{-\mathrm{T}} \mathbf{e}_{3} & =-\frac{h}{2} \zeta^{-1} \mathbf{K}_{3}^{-\mathrm{T}} \nabla a_{3}+\left(\zeta^{-1}+\frac{h^{2}}{4} \zeta^{-2} \mathbf{b}^{\mathrm{T}} \mathbf{K}_{3}^{-\mathrm{T}} \nabla a_{3}\right) \mathbf{e}_{3}+O\left(h^{2}\right) .
\end{aligned}
$$

Note that the two terms in the last expression are mutually orthogonal. Hence we obtain

$$
\left|(\mathbf{I}+\mathbf{L})^{-\mathrm{T}} \mathbf{e}_{3}\right|=\zeta^{-1}\left[1+\frac{h^{2}}{2} \zeta^{-1} \mathbf{b}^{\mathrm{T}} \mathbf{K}_{3}^{-\mathrm{T}} \nabla a_{3}+\frac{h^{2}}{4}\left|\mathbf{K}_{3}^{-\mathrm{T}} \nabla a_{3}\right|^{2}\right]^{1 / 2}+O\left(h^{2}\right),
$$

and a Taylor expansion then gives

$$
\left|(\mathbf{I}+\mathbf{L})^{-\mathrm{T}} \mathbf{e}_{3}\right|=\zeta^{-1}\left[1+\frac{h^{2}}{4} \zeta^{-1} \mathbf{b}^{\mathrm{T}} \mathbf{K}_{3}^{-\mathrm{T}} \nabla a_{3}+\frac{h^{2}}{8}\left|\mathbf{K}_{3}^{-\mathrm{T}} \nabla a_{3}\right|^{2}\right]+O\left(h^{2}\right) .
$$

Equation (114) is then obtained from (113) by direct expansion, followed by the use of (110) and (111) to eliminate $a_{3}$ and $c_{3}$. 


\section{Acknowledgement}

This research is sponsored by the National Natural Science Foundation of China (Grant nos. 11372212, 11172201) and the China Scholarship Council. The first author (YBF) thanks Professor Massimiliano Gei of Cardiff University for some very helpful discussions. The authors thank the reviewers and Professor Michel Destrade for their constructive comments that led to the statements immediately following equations (88) and (100).

\section{References}

[1] K. Bertoldi, M. Gei, Instabilities in multilayered soft dielectrics. J. Mech. Phys.. Solids 59 (2011) 18-42.

[2] J. Blok, D.G. LeGrand, Dielectric breakdown of polymer films. J. Appl. Phys. 40 (1969) 288-293.

[3] R. Bustamante, A. Dorfmann, R.W. Ogden, Nonlinear electroelastostatics: a variational framework. Z. Angew. Math. Phys. (ZAMP) 60 (2009) 154-177.

[4] R. Bustamante, J. Merodio, On weak formulations and their second variation in nonlinear electroelasticity. Mech. Res. Comm. 46 (2012) $15-19$.

[5] H.-H. Dai, Z.L. Song, On a consistent finite-strain plate theory based on three-dimensional energy principle. Proc. Roy. Soc. Lond. A 470 (2014) 20140494.

[6] G. deBotton, L. Tevet-Deree, E.A. Socolsky, Electroactive heterogeneous polymers: analysis and applications to laminated composites. Mech. Adv. Mater. Struc. 14 (2007) 13-22.

[7] M. Destrade, Y.B. Fu, A. Nobili, Edge wrinkling in elastically supported pre-stressed incompressible isotropic plates. Proc. Roy. Soc. A 472 (2016) 20160410.

[8] D. De Tommasi, G. Puglisi, G. Saccomandi, G. Zurlo, Pull-in and wrinkling instabilities of electroactive dielectric actuators, J. Phys. D: Appl. Phys. 43 (2010) 325501. 
[9] D. De Tommasi, G. Puglisi, G. Zurlo, Compression-induced failure of electroactive polymeric thin films, Appl. Phys. Lett. 98 (2011) 123507.

[10] D. De Tommasi, G. Puglisi, G. Zurlo, Electromechanical instability and oscillating deformations in electroactive polymer films, Appl. Phys. Lett. 102 (2013) 011903.

[11] D. De Tommasi, G. Puglisi, G. Zurlo, Inhomogeneous deformations and pull-in instability in electroactive polymeric films, Int. J. Non-Lin. Mech. 57 (2013) 123-129.

[12] R. Díaz-Calleja, E. Riande, M.J. Sanchis, On electromechanical stability of dielectric elastomers. Appl. Phys. Lett. 93 (2008) 101902.

[13] R. Díaz-Calleja, M.J. Sanchis, E. Riande, Effect of an electric field on the bifurcation of a biaxially stretched incompressible slab rubber. Eur. Phys. J. E 30 (2009) 417-426.

[14] R. Díaz-Calleja, M.J. Sanchis, E. Riande, Effect of an electric field on the deformation of incompressible rubbers: bifurcation phenomena. J. Electrostat. 67 (2009) 158-166.

[15] R. Díaz-Calleja, P. Llovera-Segovia, J.J. Dominguez, M.C. Rosique, A.Q. López, Theoretical modelling and experimental results of electromechanical actuation of an elastomer. J. Phys. D: Appl. Phys. 46 (2013) 235305.

[16] A. Dorfmann, R.W. Ogden, Nonlinear electroelasticity. Acta Mech. 174 (2005) 167-183.

[17] A. Dorfmann, R.W. Ogden, Nonlinear electroelasticity: incremental equations and stability. Int. J. Eng. Sci. 48 (2010) 1-14.

[18] L. Dorfmann, R.W. Ogden, Instabilities of an electroelastic plate. Int. J. Eng. Sci. 77 (2014) 79-101.

[19] L. Dorfmann, R.W. Ogden, Nonlinear Theory of Electroelastic and Magnetoelastic Interactions, Springer-Verlag (2014).

[20] L. Dorfmann, R.W. Ogden, Nonlinear electroelasticity: materials, continuum theory and applications. Proc. R. Soc. A 473 (2017) 20170311. 
[21] Y.B. Fu, L. Dorfmann, Y.-X. Xie, Necking of a dielectric membrane. Extreme Mechanics Letters 21 (2018) 44-48.

[22] M. Gei, S. Colonelli, R. Springhetti, The role of electrostriction on the stability of dielectric elastomer actuators. Int. J. Solids Struct. 51 (2013) 848-860.

[23] R. Huang, Z. Suo, Electromechanical phase transition in dielectric elastomers. Proc. R. Soc. A 468 (2012) 1014-1040.

[24] W. Hong, Modeling viscoelastic dielectrics. J. Mech. Phys. Solids 59 (2011) 637-650.

[25] J.D. Kaplunov, E.V. Nolde, G.A. Rogerson, A low-frequency model for dynamic motion in pre-stressed incompressible elastic structures. Proc. R. Soc. A 456 (2000) 2589C2610.

[26] A. Kovetz, Electromagnetic Theory, University Press, Oxford (2000).

[27] L.D. Landau, E.M. Lifshitz, Electrodynamics of continuous media, 2nd edn. Pergamon, Oxford (1984).

[28] J. Leng, L. Liu, Y. Liu, K. Yu, S. Sun, Electromechanical stability of dielectric elastomer Appl. Phys. Lett. 94 (2009) 211901.

[29] Y. Liu, L. Liu, S. Sun, J. Leng, Electromechanical stability of a Mooney-Rivlin-type dielectric elastomer with nonlinear variable permittivity, Polym. Int. 59 (2010) 371-377.

[30] R.M. McMeeking, C.M. Landis, Electrostatic forces and stored energy for deformable dielectric materials. J. Appl. Mech. 72 (2005) 581-590.

[31] M. Moscardo, X. Zhao, Z. Suo, Y. Lapusta, On designing dielectric elastomer actuators. J. Appl. Phys. 104 (2008) 093503.

[32] A.N. Norris, Comment on Method to analyze electromechanical stability of dielectric elastomers [Appl. Phys. Lett. 91, 061921 (2007)]. Appl. Phys. Lett. 92 (2008) 026101.

[33] A.V. Pichugin, G.A. Rogerson, An asymptotic membrane-like theory for long-wave motion in a pre-stressed elastic plate. Proc. R. Soc. Lond. A 458 (2002) 1447C1468. 
[34] J.S. Plante, S. Dubowsky, Large-scale failure modes of dielectric elastomer actuators. Int. J. Solids Struct. 43 (2006) 7727-7751.

[35] G. Puglisi, G. Zurlo, Electric field localizations in thin dielectric films with thickness non-uniformities. J. Electrostat. 70 (2012) 312-316.

[36] S. Rudykh, G. deBotton, Stability of anisotropic electroactive polymers with application to layered media. Z. Angew. Math. Phys. (ZAMP) 62 (2011) 1131-1142.

[37] S. Rudykh, K. Bhattacharya, G. deBotton, Snap-through actuation of thick-wall electroactive balloons. Int. J. Non-Linear Mech. 47 (2012) 206-209.

[38] S. Rudykh, K. Bhattacharya, G. deBotton, Multiscale instabilities in soft heterogeneous dielectrics. Proc. R. Soc. A 470 (2014) 20130618.

[39] K.H. Stark, C.G. Garton, Electric strength of irradiated polythene. Nature 176 (1955) 1225-1226.

[40] D. Steigmann, Two-dimensional models for the combined bending and stretching of plates and shells based on three-dimensional linear elasticity. Int. J. Eng. Sci. 46 (2008) 654-676.

[41] D. Steigmann, A well-posed finite-strain model for thin elastic sheets with bending stiffness. Math. Mech. Solids 18 (2012) 103-112.

[42] D. Steigmann, Koiter's shell theory from the perspective of threedimensional nonlinear elasticity. J. Elasticity 111 (2013) 91-107.

[43] D. Steigmann, R.W. Ogden, Classical plate buckling theory as the small-thickness limit of three-dimensional incremental elasticity. Z. Angew. Math. Mech.(ZAMM) 94 (2014) 7-20.

[44] Y.P. Su, H.C. Broderick, W.Q. Chen, M. Destrade, Wrinkles in soft dielectric plates. J. Mech. Phys. Solids 119 (2018) 298-318.

[45] Z. Suo, Theory of dielectric elastomers. Acta Mech. Solida Sin. 23 (2010) 549-578. 
[46] J. Wang, Z.L. Song, H.-H. Dai, On a consistent finite-strain plate theory for incompressible hyperelastic materials. Int. J. Solids Struct. 78-79 (2016) 101-109.

[47] Y.X. Xie, J.-C. Liu, Y.B. Fu, Bifurcation of a dielectric elastomer balloon under pressurized inflation and electric actuation. Int. J. Solids Struct. 78 (2016) 182-188.

[48] B.X. Xu, R. Mueller, M. Klassen, D. Gross, On electromechanical stability analysis of dielectric elastomer actuators. Appl. Phys. Lett. 97 (2010) 162908.

[49] X. Zhao, W. Hong, Z. Suo, Electromechanical hysteresis and coexistent states in dielectric elastomers. Phys. Rev. B 76 (2007) 134113.

[50] X. Zhao, Z. Suo, Method to analyze electromechanical stability of dielectric elastomers, Appl. Phys. Lett. 91 (2007) 061921.

[51] X. Zhao, S.J. A. Koh, Z. Suo, Nonequilibrium thermodynamics of dielectric elastomers, Int. J. App. Mech. 3 (2011) 203-217.

[52] X. Zhao, Q. Wang, Harnessing large deformation and instabilities of soft dielectrics: theory, experiment, and application. Appl. Phys. Rev. 1 (2014) 021304.

[53] J. Zhu, H. Stoyanov, G. Kofod, Z. Suo, Large deformation and electromechanical instability of a dielectric elastomer tube actuator, J. Appl. Phys. 108 (2010) 074113.

[54] G. Zurlo, Non-local elastic effects in electroactive polymers. Int. J. Nonlin. Mech. 56 (2013) 115-122.

[55] G. Zurlo, M. Destrade, D. DeTommasi, G. Puglisi, Catastrophic thinning of dielectric elastomers. Phys. Rev. Lett. 118 (2017) 078001. 